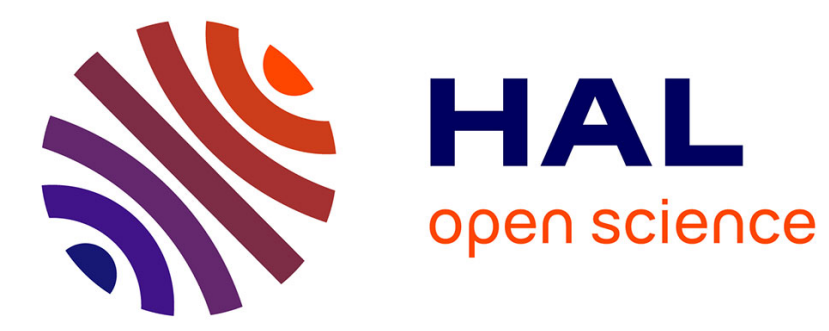

\title{
Projection schemes for fluid flows through a porous interface
}

Alfonso Caiazzo, Miguel Angel Fernández, Jean-Frédéric Gerbeau, Vincent Martin

\section{- To cite this version:}

Alfonso Caiazzo, Miguel Angel Fernández, Jean-Frédéric Gerbeau, Vincent Martin. Projection schemes for fluid flows through a porous interface. SIAM Journal on Scientific Computing, 2011, 33 (2), pp.541-564. 10.1137/100788124 . inria-00462103

\section{HAL Id: inria-00462103 https://hal.inria.fr/inria-00462103}

Submitted on 8 Mar 2010

HAL is a multi-disciplinary open access archive for the deposit and dissemination of scientific research documents, whether they are published or not. The documents may come from teaching and research institutions in France or abroad, or from public or private research centers.
L'archive ouverte pluridisciplinaire $\mathbf{H A L}$, est destinée au dépôt et à la diffusion de documents scientifiques de niveau recherche, publiés ou non, émanant des établissements d'enseignement et de recherche français ou étrangers, des laboratoires publics ou privés. 


\section{N R I A}

INSTITUT NATIONAL DE RECHERCHE EN INFORMATIQUE ET EN AUTOMATIQUE

\section{Projection schemes for fluid flows through a porous interface}

Alfonso Caiazzo — Miguel A. Fernández — Jean-Frédéric Gerbeau — Vincent Martin

$\mathbf{N}^{\circ} \mathbf{7 2 2 5}$

March 2010

Thème $\mathrm{BIO}$

apport

de recherche 



\title{
RIN RIA
}

\section{Projection schemes for fluid flows through a porous interface}

\author{
Alfonso Caiazzd*, Miguel A. Fernánded卂, Jean-Frédéric Gerbeau用, \\ Vincent Martin \\ Thème BIO - Systèmes biologiques \\ Projet REO \\ Rapport de recherche $\mathrm{n}^{\circ} 7225$ - March 2010 - 28 pages
}

\begin{abstract}
This paper presents a numerical method to simulate an incompressible fluid through an immersed porous interface. The interface is modeled by a surface measure term in the Navier-Stokes equations and it is characterized by a resistance parameter. This approach can be used for example to model valves or to simulate blood flood through an immersed stent. Starting from a monolithic formulation proposed recently, a fractional step algorithm is derived. The difficult point is that this formulation is singular when the resistance vanishes, which can be a serious issue in some applications. We show that an appropriate Nitsche's treatment of the interface condition fixes this problem and ensures uniform energy stability in time, for any non-negative value of the resistance. The theoretical stability and convergence results are illustrated with numerical experiments.
\end{abstract}

Key-words: Projection methods for Navier Stokes equations, porous interface, Nitsche's method, stabilized finite element, blood flow, stent.

* INRIA, REO project-team, Alfonso.Caiazzo@inria.fr.

$\dagger$ INRIA, REO project-team, Miguel.Fernandez@inria.fr.

¥ INRIA, REO project-team, Jean-Frederic.Gerbeau@inria.fr.

$\S$ UTC Compiègne and INRIA, REO project-team, Vincent.Martin@utc.fr.

Unité de recherche INRIA Rocquencourt

Domaine de Voluceau, Rocquencourt, BP 105, 78153 Le Chesnay Cedex (France) Téléphone : +33139635511 — Télécopie : +33139635330 


\section{Schémas de projection pour des écoulements à travers une interface poureuse}

Résumé : Dans cet article, nous présentons une méthode numérique pour simuler l'écoulement incompressible d'un fluide à travers une interface poreuse immergée dans le fluide. L'interface est modélisée comme un terme de mesure surfacique dans les équations de Navier-Stokes, faisant intervenir un paramètre appelé résistance. Cette approche peut par exemple être employée pour modéliser des valves ou pour simuler des écoulements sanguins au travers d'un stent immergé. Partant d'une formulation monolithique proposée récemment, un algorithme à pas fractionnaire est mis en place. Le problème est difficile en cela que la formulation devient singulière quand la résistance est nulle, ce qui est une sérieuse limitation pour certaines applications. Nous montrons qu'une pénalisation à la Nitsche adéquate des conditions d'interface règle ce problème. De plus ce traitement assure la stabilité en temps de l'algorithme, uniforme pour toute valeur positive ou nulle de la résistance.La stabilité théorique et les résultats de convergence sont illustrés par des expériences numériques.

Mots-clés : Méthodes de projection pour les équations de Navier-Stokes, interface poreuse, méthode de Nitsche, éléments finis stabilisés, écoulement sanguin, stent. 


\section{Introduction}

This work is devoted to the numerical simulation of an incompressible fluid through a porous immersed interface. One motivation is the modeling of the haemodynamics in aneurysms, after the implantation of a small tubular device called $a$ stent. The stent under considerations is supposed to be thin enough to be modeled as a macroscopic resistive interface term (see [1] for the homogenization of sieve problems). Another application is a simplified model of heart valves recently proposed in 2. In this case, the resistance of the immersed interface is very large when the valve is closed and vanishes when the valve is open. It is therefore important to keep in mind that the numerical method has to be robust in these two extreme regimes.

In 10, this immersed porous interface model was presented and analyzed in the case of the stationary Stokes equations using finite elements of equal order for the velocity and the pressure. In the present paper we show how this model can be implemented in a projection method. Interestingly, the immersed porous interface model introduces a singularity in the projection step when the resistance of the interface vanishes. The main goal of this paper is to propose a numerical method to circumvent this singularity.

Projection methods for the incompressible Navier-Stokes equations, as originally introduced in [7, 17, are fractional step schemes which consist in splitting the time evolution into two sub-steps. First, an intermediate velocity, that does not fulfill the incompressibility constraint, is computed solving an advectiondiffusion problem. Second, the final velocity and pressure are obtained by orthogonally projecting the intermediate velocity onto a divergence-free space. Projection methods are very efficient at solving the incompressible Navier-Stokes equations and have been widely used and analyzed for four decades. We refer to 12 for a recent review, and to 4 for a convergence analysis using equal order pressure/velocity approximations. Projection schemes have been recently shown to be specially appealing for fluid-structure interaction problems. Indeed, the fractional step formulation of the fluid problem can be used to decompose the coupling strategy, allowing for a semi-implicit fluid-structure coupling 3 , 9 (see also 16 ). This has been shown to be much more efficient than any fully implicit coupling, without compromising stability.

To design a projection scheme for the porous interface model, the transmission conditions through the interface have to be carefully split in the two sub-steps. When the projection step is solved through a Darcy problem, the formulation is only a straightforward extension of the one proposed in 10 . But when a Poisson formulation of the projection is chosen, some terms of the equation are divided by the resistance coefficient of the porous interface. The formulation is therefore singular for a vanishing resistance. This singularity, which is a pure artifact of the formulation, could be trivially circumvented by taking a "very small" resistance instead of a zero one. But, as will be shown in our numerical simulations, this results in a poorly conditioned problem and increases dramatically the number of iterations needed to solve the projection step with an iterative method. As mentioned above, this issue is striking for the simplified heart valve simulations of [2] since a zero resistance is actually used to model open valves. In addition, for semi-implicit fluid-structure algorithms, the projection step is precisely the only one which is implicitly coupled to the 
structure. It is therefore critical to solve it very efficiently, for any values of the resistance.

We present in this paper a method that is valid in the limit case of a completely permeable interface (zero resistance) and gives better conditioned linear systems in the regime of very small resistances. The algorithm is based on a modified formulation of the pressure problem including a stabilization term $\grave{a} l a$ Nitsche [15, 5, 13. It is inspired by a recent idea proposed in 14 to deal with Dirichlet and Neumann boundary conditions in a unified formulation.

The article is organized as follows. In $\$ 2$, the porous interface model is briefly described and the main notation is introduced. Section 3 contains the main results of the article. The method is derived in 3.2 and the non-stabilized algorithm is written in $\$ 3.3$. In $\$ 3.4$ the stabilization $\dot{a}$ la Nitsche is introduced and analyzed in the stationary case. The stabilized discrete projection scheme is given in 33.5 Finally, 33.6 is devoted to the proof of the stability in time of the projection methods, with and without stabilization. Numerical validations of the schemes are shown in 8 . Section 5 sums up the main results and draws some conclusions.

\section{Incompressible fluid through a porous inter- face}

We consider an incompressible fluid governed by the transient Navier-Stokes equations in a smooth domain $\Omega \subset \mathbb{R}^{d}, d=2$ or 3 , during the time interval $(0, T)$. A porous interface is assumed to occupy an hyperplane $\Gamma \subset \mathbb{R}^{d-1}$ which divides the fluid domain in two connected subdomains (see Figure 1), that is,

$$
\Omega=\Omega_{1} \cup \Gamma \cup \Omega_{2}, \quad \Sigma_{i} \stackrel{\text { def }}{=} \partial \Omega \cap \bar{\Omega}_{i}, i=1,2 .
$$

In $\Omega_{1}$ (resp. $\Omega_{2}$ ), the outward normal at the interface is denoted by $\boldsymbol{n}_{1}$ (resp. $\boldsymbol{n}_{2}$ ). We also introduce the notation $\boldsymbol{n} \stackrel{\text { def }}{=} \boldsymbol{n}_{1}=-\boldsymbol{n}_{2}$.

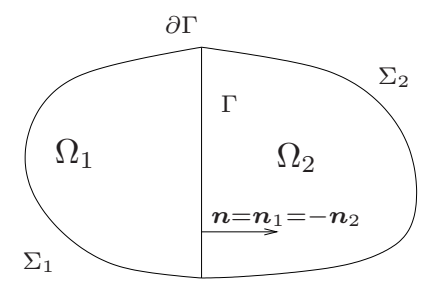

Figure 1: A domain $\Omega$ decomposed into two subdomains $\Omega_{1}$ and $\Omega_{2}$, separated by the hyperplane $\Gamma$.

The porous interface introduces an additional dissipative term in the momentum equation. Thus, the fluid velocity $\boldsymbol{u}$ and pressure $p$ are driven by the following modified Navier-Stokes equations (see [10]):

$$
\begin{aligned}
\rho_{f}\left(\partial_{t} \boldsymbol{u}+\boldsymbol{u} \cdot \boldsymbol{\nabla u}\right)+\nabla p-2 \mu \operatorname{div}(\boldsymbol{\epsilon}(\boldsymbol{u}))+r_{\Gamma} \boldsymbol{u} \delta_{\Gamma}=\boldsymbol{f} & \text { in } \quad \Omega \\
\operatorname{div} \boldsymbol{u}=0 & \text { in } \quad \Omega \\
\boldsymbol{u}=\mathbf{0} & \text { on } \quad \partial \Omega,
\end{aligned}
$$


where $\rho_{f}$ denotes the fluid density, $\mu$ the fluid viscosity, $\boldsymbol{\epsilon}(\boldsymbol{u}) \stackrel{\text { def }}{=} 1 / 2\left(\boldsymbol{\nabla} \boldsymbol{u}+\boldsymbol{\nabla} \boldsymbol{u}^{T}\right)$ the strain rate tensor, $\boldsymbol{f}$ a given volume force, $\delta_{\Gamma}$ the Dirac measure on $\Gamma$, and $r_{\Gamma}$ a given interface resistance, related to the permeability and porosity of the interface. Without loss of generality, here we have assumed that $r_{\Gamma}$ is simply a non-negative scalar. The analysis below can however be extended, with minor modifications, to the case in which $r_{\Gamma}$ is replaced by a symmetric positive semi-definite tensor (see [10]). Moreover, we assume that homogeneous Dirichlet boundary conditions are enforced on $\partial \Omega$.

For any field $q$ defined in $\Omega$, let us consider the notation $\left.q_{i} \stackrel{\text { def }}{=} q\right|_{\Omega_{i}}$ for the restriction of $q$ to $\Omega_{i}(i=1,2)$. We then define the following jumps across $\Gamma$ :

$$
\begin{aligned}
\llbracket \boldsymbol{u} \rrbracket & \stackrel{\text { def }}{=} \boldsymbol{u}_{1 \mid \Gamma}-\boldsymbol{u}_{2 \mid \Gamma}, \\
\llbracket \boldsymbol{\epsilon}(\boldsymbol{u}) \boldsymbol{n} \rrbracket & \stackrel{\text { def }}{=} \boldsymbol{\epsilon}\left(\boldsymbol{u}_{1}\right)_{\mid \Gamma} \boldsymbol{n}_{1}+\boldsymbol{\epsilon}\left(\boldsymbol{u}_{2}\right)_{\mid \Gamma} \boldsymbol{n}_{2}, \\
\llbracket p \boldsymbol{n} \rrbracket & \stackrel{\text { def }}{=} p_{1 \mid \Gamma} \boldsymbol{n}_{1}+p_{2 \mid \Gamma} \boldsymbol{n}_{2} .
\end{aligned}
$$

Problem (11) can be reformulated equivalently as the following two-domain coupled problem, in terms of $\boldsymbol{u}_{i}$ and $p_{i}$ :

$$
\begin{aligned}
\rho_{f}\left(\partial_{t} \boldsymbol{u}_{i}+\boldsymbol{u}_{i} \cdot \boldsymbol{\nabla} \boldsymbol{u}_{i}\right)+\nabla p_{i}-2 \mu \operatorname{div}\left(\boldsymbol{\epsilon}\left(\boldsymbol{u}_{i}\right)\right)=\boldsymbol{f}_{i} & \text { in } \quad \Omega_{i}, \\
\operatorname{div} \boldsymbol{u}_{i}=0 & \text { in } \quad \Omega_{i}, \\
\boldsymbol{u}_{i}=\mathbf{0} & \text { on } \quad \Sigma_{i},
\end{aligned}
$$

complemented with the interface conditions

$$
\begin{array}{rll}
\llbracket \boldsymbol{u} \rrbracket=\mathbf{0} & \text { on } & \Gamma, \\
\llbracket 2 \mu \boldsymbol{\epsilon}(\boldsymbol{u}) \boldsymbol{n}-p \boldsymbol{n} \rrbracket=-r_{\Gamma} \boldsymbol{u} & \text { on } & \Gamma .
\end{array}
$$

These interface transmission conditions enforce the continuity of the velocity and relate the stress jump across the interface to the velocity.

\subsection{Preliminaries}

Let $\mathcal{O}$ be a bounded open set in $\mathbb{R}^{p}(d-1 \leq p \leq d)$. We consider the usual Sobolev spaces $H^{m}(\mathcal{O})$, for $m \geq 0$. In particular, $L^{2}(\mathcal{O})=H^{0}(\mathcal{O})$. We denote by $(\cdot, \cdot)_{\mathcal{O}}$ the scalar product in $L^{2}(\mathcal{O})$ and by $\|\cdot\|_{0, \mathcal{O}}$ the corresponding norm. We will often omit the subscript $\mathcal{O}$ in the case $\mathcal{O}=\Omega$.

We denote by $H_{0}^{1}(\mathcal{O})$ the subspace of $H^{1}(\mathcal{O})$ consisting of functions vanishing on $\partial \mathcal{O}$, and by $L_{0}^{2}(\mathcal{O})$ the subspace of $L^{2}(\mathcal{O})$ consisting of functions with zero mean in $\mathcal{O}$.

We introduce the spaces

$$
\boldsymbol{V} \stackrel{\text { def }}{=}\left[H_{0}^{1}(\Omega)\right]^{d}, \quad Q \stackrel{\text { def }}{=} L_{0}^{2}(\Omega), \quad M_{i} \stackrel{\text { def }}{=} H^{1}\left(\Omega_{i}\right) \quad i=1,2,
$$

and

$$
M \stackrel{\text { def }}{=}\left\{v \in L^{2}(\Omega) \mid v_{i} \in M_{i} \quad i=1,2\right\}, \quad N \stackrel{\text { def }}{=} M \cap Q .
$$

To define a finite element approximation, we introduce a regular family of triangulations $\left\{\mathcal{T}_{h}\right\}_{0<h \leq 1}$ of $\Omega$, in the standard sense of $[\underline{8}$. The level of refinement of the triangulation $\mathcal{T}_{h}$ is defined by

$$
h=\max _{T \in \mathcal{T}_{h}} h_{T},
$$


$h_{T}$ being the diameter of the element $T$. For the sake of simplicity, we assume that all the considered triangulations are quasi-uniform, that is, there exist two positive constants $C_{\min }$ and $C_{\max }$ such that

$$
C_{\min } h_{T} \leq h \leq C_{\max } h_{T}, \quad \forall T \in \mathcal{T}_{h}, \quad \forall 0<h \leq 1 .
$$

Moreover we assume that, for all $h \in(0,1]$, the triangulation $\mathcal{T}_{h}$ is conforming with the interface $\Gamma$. Hence, we denote by $\mathcal{G}_{h}$ the corresponding triangulation of the interface. Moreover, for each edge $(d=2)$ or face $(d=3) E \in \mathcal{G}_{h}$, we denote by $h_{E}$ its diameter.

We now introduce the finite element spaces of degree $k \geq 1, \boldsymbol{V}_{h}^{k}$ and $N_{h}^{k}$ equal order approximations of $\boldsymbol{V}$ and $Q$, as follows:

$$
\begin{aligned}
\boldsymbol{V}_{h}^{k} \stackrel{\text { def }}{=}\left\{\boldsymbol{v}_{h} \in\left(\mathcal{C}^{0}(\bar{\Omega})\right)^{d} \mid \boldsymbol{v}_{h \mid T} \in\left(\mathbb{P}_{k}\right)^{d} \forall T \in \mathcal{T}_{h}\right\} \cap \boldsymbol{V}, \\
M_{h, i}^{k} \stackrel{\text { def }}{=}\left\{q_{h} \in \mathcal{C}^{0}\left(\bar{\Omega}_{i}\right) \mid q_{h \mid T} \in \mathbb{P}_{k} \quad \forall T \in \mathcal{T}_{h}\right\} \quad i=1,2, \\
M_{h}^{k} \stackrel{\text { def }}{=}\left\{q_{h} \in L^{2}(\Omega) \mid q_{h \mid \Omega_{i}} \in M_{h, i}^{k} \quad i=1,2\right\}, \\
N_{h}^{k} \stackrel{\text { def }}{=} M_{h}^{k} \cap Q .
\end{aligned}
$$

Note that the discrete pressure, looked for into $N_{h}^{k}$, can be discontinuous across the interface $\Gamma$. This is of utmost importance to get a correct approximation of the solution without refining the mesh exceedingly, as underlined in [10].

In the analysis below we shall make use of the following trace-inverse inequality (see, e.g., [6, 18])

$$
\sum_{E \in \mathcal{G}_{h}} h_{E}\left\|q_{h, i}\right\|_{0, E}^{2} \leq C_{\mathrm{T}}\left\|q_{h, i}\right\|_{0, \Omega_{i}}^{2}, \quad i=1,2
$$

for all $q_{h} \in M_{h}^{k}$ and where $C_{\mathrm{T}}>0$ is a constant independent of $h$ (but which might depend on $k$ ).

Let $N_{T} \in \mathbb{N}^{*}$ be a given integer and consider a uniform partition $\left\{\left[t_{n}, t_{n+1}\right]\right\}_{0 \leq n \leq N_{T}-1}$, with $t_{n} \stackrel{\text { def }}{=} n \delta t$, of the time interval of interest $(0, T)$, with time-step $\delta t \stackrel{\text { def }}{=} T / N_{T}$. For a given time dependent function $X$, the quantity $X^{n}$ denotes an approximation of $X\left(t_{n}\right)$ and we set $\boldsymbol{f}^{n} \stackrel{\text { def }}{=} \boldsymbol{f}\left(\cdot, t_{n}\right)$.

Finally, for a given vector field $\boldsymbol{v}$, we denote by $v_{\boldsymbol{n}}$ its normal component.

\section{Projection method for porous interface prob- lems}

In this section, we derive and analyze a projection-based method for the numerical approximation of (11). For the sake of conciseness, we shall omit the nonlinear term $\boldsymbol{u} \cdot \boldsymbol{\nabla} \boldsymbol{u}$ in the discussion below. The proposed algorithms can be adapted straightforwardly to the non-linear case, for instance, by performing a semi-implicit discretization of the nonlinear term.

We first briefly recall the original Chorin-Temam projection scheme [7, 17. In practice, it is advocated to rather consider variants of this scheme which fix some precision issues, for example the incremental pressure correction 11] or the rotational incremental pressure correction [19]. We refer to [12] for a 
review of the possible choices. The ideas presented here can be readily extend to those variants. So, for simplicity, we limit the presentation to the original Chorin-Temam algorithm.

The projection algorithm consists of solving the Navier-Stokes equations in two steps. In the viscous step, we search for a velocity field $\widetilde{\boldsymbol{u}}^{n+1}$ solution of the diffusion problem

$$
\begin{array}{r}
\frac{\rho_{f}}{\delta t}\left(\widetilde{\boldsymbol{u}}^{n+1}-\boldsymbol{u}^{n}\right)-2 \mu \operatorname{div}\left(\boldsymbol{\epsilon}\left(\widetilde{\boldsymbol{u}}^{n+1}\right)\right)=\boldsymbol{f}^{n+1} \quad \text { in } \quad \Omega, \\
\widetilde{\boldsymbol{u}}^{n+1}=\mathbf{0} \quad \text { on } \quad \partial \Omega .
\end{array}
$$

Of course, in practice, an advection term is also added to this step. Next, a divergence free velocity $\boldsymbol{u}^{n+1}$ and the pressure $p^{n+1}$ are recovered by projecting $\widetilde{\boldsymbol{u}}^{n+1}$ onto a divergence-free velocity space, which reads:

$$
\begin{aligned}
\frac{\rho_{f}}{\delta t}\left(\boldsymbol{u}^{n+1}-\widetilde{\boldsymbol{u}}^{n+1}\right)+\boldsymbol{\nabla} p^{n+1} & =\mathbf{0} \text { in } \Omega, \\
\operatorname{div} \boldsymbol{u}^{n+1}=0 & \text { in } \Omega, \\
\boldsymbol{u}^{n+1} \cdot \boldsymbol{n} & =0 \text { on } \partial \Omega .
\end{aligned}
$$

The end-of-step velocity $\boldsymbol{u}^{n}$ can be eliminated in (8) by noting that, from (9),

$$
\frac{\rho_{f}}{\delta t} \boldsymbol{u}^{n}=\frac{\rho_{f}}{\delta t} \widetilde{\boldsymbol{u}}^{n}-\nabla p^{n}
$$

and, hence, (8) becomes

$$
\begin{aligned}
& \frac{\rho_{f}}{\delta t}\left(\widetilde{\boldsymbol{u}}^{n+1}-\widetilde{\boldsymbol{u}}^{n}\right)-2 \mu \operatorname{div}\left(\boldsymbol{\epsilon}\left(\widetilde{\boldsymbol{u}}^{n+1}\right)\right)+\nabla p^{n}=\boldsymbol{f}^{n+1} \quad \text { in } \Omega, \\
& \widetilde{\boldsymbol{u}}^{n+1}=\mathbf{0} \text { on } \partial \Omega \text {. }
\end{aligned}
$$

Moreover, under suitable regularity assumptions, we can take the divergence of the first equation in (9), which leads to:

$$
\begin{aligned}
-\Delta p^{n+1}=-\frac{\rho_{f}}{\delta t} \operatorname{div} \widetilde{\boldsymbol{u}}^{n+1} & \text { in } \quad \Omega, \\
\partial_{\boldsymbol{n}} p^{n+1}=0 & \text { on } \quad \partial \Omega .
\end{aligned}
$$

System (8)-(9) corresponds to the Darcy formulation of the projection scheme, whereas (11)-(12) is the pressure-Poisson formulation of the projection scheme. In this paper, we will mainly focus on the latter, which is generally preferred for efficiency reasons.

\subsection{A domain-decomposition point of view}

The key point on the derivation of a projection scheme for (1) lies on how the interface conditions (3)-(4) are split in time. To this aim it is useful to identify how these conditions split for the case $r_{\Gamma}=0$, which corresponds to the domaindecomposition formulation of the standard projection scheme (11)-(12). In this case, problem (11) is equivalent to solving (for $i=1,2$ )

$$
\begin{array}{r}
\frac{\rho_{f}}{\delta t}\left(\widetilde{\boldsymbol{u}}_{i}^{n+1}-\widetilde{\boldsymbol{u}}_{i}^{n}\right)-2 \mu \operatorname{div}\left(\boldsymbol{\epsilon}\left(\widetilde{\boldsymbol{u}}_{i}^{n+1}\right)\right)+\boldsymbol{\nabla} p_{i}^{n}=\boldsymbol{f}_{i}^{n+1} \text { in } \quad \Omega_{i}, \\
\widetilde{\boldsymbol{u}}_{i}^{n+1}=\mathbf{0} \quad \text { on } \quad \Sigma_{i},
\end{array}
$$


with the interface conditions

$$
\begin{aligned}
\llbracket \widetilde{\boldsymbol{u}}^{n+1} \rrbracket & =\mathbf{0} \quad \text { on } \quad \Gamma, \\
\llbracket 2 \mu \boldsymbol{\epsilon}\left(\widetilde{\boldsymbol{u}}^{n+1}\right) \boldsymbol{n}-p^{n} \boldsymbol{n} \rrbracket & =\mathbf{0} \quad \text { on } \quad \Gamma,
\end{aligned}
$$

and, similarly, problem (12) can be reformulated as

$$
\begin{array}{r}
-\Delta p_{i}^{n+1}=-\frac{\rho_{f}}{\delta t} \operatorname{div} \widetilde{\boldsymbol{u}}_{i}^{n+1} \quad \text { in } \quad \Omega_{i}, \\
\partial_{\boldsymbol{n}} p_{i}^{n+1}=0 \quad \text { on } \quad \Sigma_{i},
\end{array}
$$

with the interface conditions

$$
\begin{aligned}
& \llbracket p^{n+1} \rrbracket=0 \quad \text { on } \quad \Gamma, \\
& \llbracket \partial_{\boldsymbol{n}} p^{n+1} \rrbracket=0 \text { on } \Gamma \text {. }
\end{aligned}
$$

\subsection{Fractional step for the immersed porous interface model}

The splitting (14)-(16) corresponds to the interface conditions (3)-(4) with $r_{\Gamma}=$ 0 . We now generalize it to the case $r_{\Gamma}>0$. Within each subdomain $\Omega_{i}$, problems (13) and (15) remain the same. On the one hand, we propose to replace (14) by

$$
\begin{array}{r}
\llbracket \widetilde{\boldsymbol{u}}^{n+1} \rrbracket=\mathbf{0} \quad \text { on } \quad \Gamma, \\
\llbracket 2 \mu \boldsymbol{\epsilon}\left(\widetilde{\boldsymbol{u}}^{n+1}\right) \boldsymbol{n}-p^{n} \boldsymbol{n} \rrbracket=-r_{\Gamma} \widetilde{\boldsymbol{u}}^{n+1} \quad \text { on } \quad \Gamma,
\end{array}
$$

which is consistent with (3)-(4). On the other hand, we replace (16) by

$$
\begin{aligned}
& \llbracket p^{n+1} \rrbracket=r_{\Gamma} \boldsymbol{u}^{n+1} \cdot \boldsymbol{n} \quad \text { on } \Gamma, \\
& \llbracket \partial_{\boldsymbol{n}} p^{n+1} \rrbracket=0 \quad \text { on } \Gamma,
\end{aligned}
$$

where the end-of-step velocity $\boldsymbol{u}^{n+1}$ can be eliminated using (10) at time level $n+1$. This yields the Robin-like interface condition

$$
\frac{r_{\Gamma} \delta t}{\rho_{f}} \partial_{\boldsymbol{n}} p^{n+1}+\llbracket p^{n+1} \rrbracket=r_{\Gamma} \widetilde{\boldsymbol{u}}^{n+1} \cdot \boldsymbol{n} \quad \text { on } \quad \Gamma .
$$

Note that $(18)_{1}$ amounts to neglecting the normal component of the viscous stress in (4), which is the usual way of treating a stress boundary condition in a projection scheme (see [12, Section 10.1]). In addition, the continuity of the normal derivative of $p^{n+1}(18)_{2}$ follows from the continuity of $\boldsymbol{u}^{n+1} \cdot \boldsymbol{n}$ and of $\widetilde{\boldsymbol{u}}^{n+1}$, as can be inferred from (10).

In summary, the proposed splitting of the interface conditions (3)-(44) for $r_{\Gamma}>0$ is given by (17) for the viscous step and by

$$
\begin{aligned}
& \frac{r_{\Gamma} \delta t}{\rho_{f}} \partial_{\boldsymbol{n}} p^{n+1}+\llbracket p^{n+1} \rrbracket=r_{\Gamma} \widetilde{\boldsymbol{u}}^{n+1} \cdot \boldsymbol{n}, \quad \text { on } \quad \Gamma, \\
& \llbracket \partial_{n} p^{n+1} \rrbracket=0 \quad \text { on } \Gamma,
\end{aligned}
$$

for the projection step. Needless to say that these conditions (17)-(19) reduce to (14)-(16) for $r_{\Gamma}=0$.

We conclude this subsection by detailing the weak formulation of (13) and (15) with the interface conditions (17) and (19). For $r_{\Gamma}>0$, the formulation reads: 
1. Viscous step: find $\widetilde{\boldsymbol{u}}^{n+1} \in \boldsymbol{V}$ such that

$$
\begin{aligned}
& \frac{\rho_{f}}{\delta t}\left(\widetilde{\boldsymbol{u}}^{n+1}, \boldsymbol{v}\right)+2 \mu\left(\boldsymbol{\epsilon}\left(\widetilde{\boldsymbol{u}}^{n+1}\right), \boldsymbol{\epsilon}(\boldsymbol{v})\right)+r_{\Gamma}\left(\widetilde{\boldsymbol{u}}^{n+1}, \boldsymbol{v}\right)_{\Gamma}= \\
& \quad\left(\boldsymbol{f}^{n+1}, \boldsymbol{v}\right)+\frac{\rho_{f}}{\delta t}\left(\widetilde{\boldsymbol{u}}^{n}, \boldsymbol{v}\right)-\sum_{i=1,2}\left(\boldsymbol{\nabla} p_{i}^{n}, \boldsymbol{v}_{i}\right)_{\Omega_{i}}+\left(\llbracket p^{n} \rrbracket, \boldsymbol{v} \cdot \boldsymbol{n}\right)_{\Gamma},
\end{aligned}
$$

for all $v \in V$.

2. Projection step $\left(r_{\Gamma}>0\right)$ : find $p^{n+1} \in N$ such that

$$
\begin{aligned}
\sum_{i=1,2}\left(\nabla p_{i}^{n+1}, \nabla q_{i}\right)_{\Omega_{i}} & +\frac{\rho_{f}}{r_{\Gamma} \delta t}\left(\llbracket p^{n+1} \rrbracket, \llbracket q \rrbracket\right)_{\Gamma} \\
& =\frac{\rho_{f}}{\delta t}\left[-\left(\operatorname{div} \widetilde{\boldsymbol{u}}^{n+1}, q\right)+\left(\widetilde{\boldsymbol{u}}^{n+1} \cdot \boldsymbol{n}, \llbracket q \rrbracket\right)_{\Gamma}\right]
\end{aligned}
$$

for all $q \in N$.

\subsection{Discrete projection scheme}

Replacing in (20) and (21) the spaces $\boldsymbol{V}$ and $N$ by the finite element approximation spaces $\boldsymbol{V}_{h}^{k}$ and $N_{h}^{k}$ defined in (6) , we get the fully discrete projection algorithm: given an initial discrete velocity $\widetilde{\boldsymbol{u}}_{h}^{0} \in \boldsymbol{V}_{h}^{k}$, an initial discrete pressure $p_{h}^{0} \in N_{h}^{k}$, solve for $n=0, \ldots, N_{T}-1$ :

1. Viscous step: find $\widetilde{\boldsymbol{u}}_{h}^{n+1} \in \boldsymbol{V}_{h}^{k}$ such that

$$
\begin{aligned}
& \frac{\rho_{f}}{\delta t}\left(\widetilde{\boldsymbol{u}}_{h}^{n+1}, \boldsymbol{v}_{h}\right)+2 \mu\left(\boldsymbol{\epsilon}\left(\widetilde{\boldsymbol{u}}_{h}^{n+1}\right), \boldsymbol{\epsilon}\left(\boldsymbol{v}_{h}\right)\right)+r_{\Gamma}\left(\widetilde{\boldsymbol{u}}_{h}^{n+1}, \boldsymbol{v}_{h}\right)_{\Gamma}= \\
& \left(\boldsymbol{f}^{n+1}, \boldsymbol{v}_{h}\right)+\frac{\rho_{f}}{\delta t}\left(\widetilde{\boldsymbol{u}}_{h}^{n}, \boldsymbol{v}_{h}\right)-\sum_{i=1,2}\left(\boldsymbol{\nabla} p_{h, i}^{n}, \boldsymbol{v}_{h, i}\right)_{\Omega_{i}}+\left(\llbracket p_{h}^{n} \rrbracket, \boldsymbol{v}_{h} \cdot \boldsymbol{n}\right)_{\Gamma},
\end{aligned}
$$

for all $\boldsymbol{v}_{h} \in \boldsymbol{V}_{h}^{k}$.

2. Projection step $\left(r_{\Gamma}>0\right)$ : find $p_{h}^{n+1} \in N_{h}^{k}$ such that

$$
\begin{aligned}
\sum_{i=1,2}\left(\nabla p_{h, i}^{n+1}, \nabla q_{h, i}\right)_{\Omega_{i}} & +\frac{\rho_{f}}{r_{\Gamma} \delta t}\left(\llbracket p_{h}^{n+1} \rrbracket, \llbracket q_{h} \rrbracket\right)_{\Gamma} \\
= & \frac{\rho_{f}}{\delta t}\left[-\left(\operatorname{div} \widetilde{\boldsymbol{u}}_{h}^{n+1}, q_{h}\right)+\left(\widetilde{\boldsymbol{u}}_{h}^{n+1} \cdot \boldsymbol{n}, \llbracket q_{h} \rrbracket\right)_{\Gamma}\right]
\end{aligned}
$$

for all $q_{h} \in N_{h}^{k}$.

\subsection{Stabilized projection via Nitsche interface method}

If $r_{\Gamma}>0$, it is straightforward to see that problem (23) admits a unique solution. However, formulation (23) is not defined for $r_{\Gamma}=0$. This singularity is not inherent to the physical problem but is rather a numerical artifact of the pressure-Poisson formulation of the projection step. For some applications we have in mind, the limitation $r_{\Gamma}>0$ is too strong. Indeed, as mentioned in the introduction, this formulation could be used in a simplified model of valves for 
which it is necessary to treat the case $r_{\Gamma}=0$ (open valves). Another situation when the resistance vanishes is the apparition of holes in a porous surface.

In those cases when $r_{\Gamma}=0$, it is possible to replace $r_{\Gamma}$ by a "very small value" in the numerical scheme. But, as will be shown in the numerical experiments, this dramatically deteriorates the efficiency of the iterative solvers. Note that more generally, this trouble occurs for positive $r_{\Gamma}$ when $\delta t_{\Gamma}$ is very small with respect to $\rho_{f}$.

In this section, we propose a new formulation of the pressure problem (23) based on a Nitsche-like approach. This formulation is consistent with the original problem, is valid for any nonnegative $r_{\Gamma}$, and has a better behavior when $\frac{\delta t r_{\Gamma}}{\rho_{f}} \ll 1$.

\subsubsection{Nitsche interface method with a resistive interface}

We present the method on the following problem: let $\alpha>0$ be a given constant, let $f_{i} \in L^{2}\left(\Omega_{i}\right), i=1,2$ and $g_{1}, g_{2} \in L^{2}(\Gamma)$ be given functions, solve

$$
\begin{array}{rll}
-\Delta p_{i}=f_{i} & \text { in } \quad \Omega_{i}, \\
\partial_{\boldsymbol{n}_{i}} p_{i}=0 & \text { on } \quad \Sigma_{i},
\end{array}
$$

for $i=1,2$, with the interface conditions

$$
\begin{aligned}
& \partial_{\boldsymbol{n}_{1}} p_{1}=\frac{1}{\alpha}\left(p_{2}-p_{1}\right)+g_{1} \quad \text { on } \quad \Gamma, \\
& \partial_{\boldsymbol{n}_{2}} p_{2}=\frac{1}{\alpha}\left(p_{1}-p_{2}\right)+g_{2} \quad \text { on } \quad \Gamma .
\end{aligned}
$$

We assume that the compatibility conditions

$$
g_{1}+g_{2}=0 \text { and } \sum_{i=1,2} \int_{\Omega_{i}} f_{i}=0
$$

hold, ensuring that problem (24)-(25) is well posed. In this case, the normal derivative of $p$ is continuous across $\Gamma$

$$
\llbracket \partial_{\boldsymbol{n}} p \rrbracket=\partial_{\boldsymbol{n}_{1}} p_{1}+\partial_{\boldsymbol{n}_{2}} p_{2}=0 \quad \text { on } \Gamma .
$$

When $\alpha$ goes to zero, $p_{1}=p_{2}$ is enforced by penalization on $\Gamma$, and the system (24)-(25) can be viewed as a domain decomposition formulation of the Laplace problem over the whole domain $\Omega$. Conversely, when $\alpha$ goes to $\infty$, the system (24)-(25) tends to two separate Neumann problems on $\Omega_{1}$ and $\Omega_{2}$.

Problem (24)-(25) is the strong counterpart of problem (23) with

$$
\alpha=\frac{r_{\Gamma} \delta t}{\rho_{f}},
$$

and

$$
f_{i}=\frac{\rho_{f}}{\delta t} \operatorname{div} \widetilde{\boldsymbol{u}}_{i}, \quad g_{i}=\frac{\rho_{f}}{\delta t} \widetilde{\boldsymbol{u}}_{i} \cdot \boldsymbol{n}_{i}, \quad \text { for } i=1,2,
$$

that satisfy (26) since $\llbracket \widetilde{\boldsymbol{u}} \rrbracket=0$ on $\Gamma$, and $\boldsymbol{u}=0$ on $\partial \Omega$.

The idea of the stabilized approach is to enforce weakly the interface conditions on $\Gamma$ using a Nitsche-like penalization. For a positive constant $\gamma$, we 
consider the following modified formulation of the pressure problem (24)-(25): find $p_{h} \in N_{h}^{k}$, such that

$$
\mathcal{C}_{h, \alpha}\left(p_{h}, q_{h}\right)=\mathcal{G}_{h, \alpha}\left(q_{h}\right),
$$

or all $q_{h} \in N_{h}^{k}$, with

$$
\begin{aligned}
\mathcal{C}_{h, \alpha}(p, q) \stackrel{\text { def }}{=} & \sum_{i=1,2}\left(\nabla p_{i}, \nabla q_{i}\right)_{\Omega_{i}}-\sum_{E \in \mathcal{G}_{h}} \frac{\gamma h_{E}}{\alpha+\gamma h_{E}}\left[\left(\partial_{\boldsymbol{n}_{1}} p_{1}, \llbracket q \rrbracket\right)_{E}+\left(\llbracket p \rrbracket, \partial_{\boldsymbol{n}_{1}} q_{1}\right)_{E}\right] \\
& +\sum_{E \in \mathcal{G}_{h}} \frac{1}{\alpha+\gamma h_{E}}(\llbracket p \rrbracket, \llbracket q \rrbracket)_{E}-\sum_{E \in \mathcal{G}_{h}} \frac{\alpha \gamma h_{E}}{\alpha+\gamma h_{E}}\left(\partial_{\boldsymbol{n}_{1}} p_{1}, \partial_{\boldsymbol{n}_{1}} q_{1}\right)_{E}, \\
\mathcal{G}_{h, \alpha}(q) \stackrel{\text { def }}{=} & \sum_{i=1,2}\left(f_{i}, q_{i}\right)_{\Omega_{i}}+\sum_{E \in \mathcal{G}_{h}} \frac{\alpha}{\alpha+\gamma h_{E}}\left(g_{1}, \llbracket q \rrbracket\right)_{E}-\sum_{E \in \mathcal{G}_{h}} \frac{\alpha \gamma h_{E}}{\alpha+\gamma h_{E}}\left(g_{1}, \partial_{\boldsymbol{n}_{1}} q_{1}\right)_{E} .
\end{aligned}
$$

The new terms in (30), compared to (23), will be made clear in the next section.

It is worth noticing that this definition is valid for $\alpha=0$. Indeed, in this case, (29) reads

$$
\begin{aligned}
\sum_{i=1,2}\left(\nabla p_{h, i}, \nabla q_{h, i}\right)_{\Omega_{i}}-\left(\partial_{\boldsymbol{n}_{1}} p_{h, 1}, \llbracket q_{h} \rrbracket\right)_{\Gamma}-\left(\llbracket p_{h} \rrbracket, \partial_{\boldsymbol{n}_{1}} q_{h, 1}\right)_{\Gamma} \\
+\sum_{E \in \mathcal{G}_{h}} \frac{1}{\gamma h_{E}}\left(\llbracket p_{h} \rrbracket, \llbracket q_{h} \rrbracket\right)_{E}=\sum_{i=1,2}\left(f_{i}, q_{h, i}\right)_{\Omega_{i}} .
\end{aligned}
$$

This is the interface Nitsche formulation introduced in 5]. It approximates the solution of the Poisson problem in $\Omega$ using discontinuous approximations across $\Gamma$ (but continuous in each $\Omega_{i}$ ).

Moreover, when $\alpha \rightarrow \infty$, the formulation (29) formally reads

$$
\begin{aligned}
\sum_{i=1,2}\left(\nabla p_{h, i},\right. & \left.\nabla q_{h, i}\right)_{\Omega_{i}}-\sum_{E \in \mathcal{G}_{h}} \gamma h_{E}\left(\partial_{\boldsymbol{n}_{1}} p_{h, 1}, \partial_{\boldsymbol{n}_{1}} q_{h, 1}\right)_{E} \\
& =\sum_{i=1,2}\left(f_{i}, q_{h, i}\right)_{\Omega_{i}}+\sum_{i=1,2}\left(g_{i}, q_{h, i}\right)_{\Gamma}-\sum_{E \in \mathcal{G}_{h}} \gamma h_{E}\left(g_{1}, \partial_{\boldsymbol{n}_{1}} q_{h, 1}\right)_{E} \cdot
\end{aligned}
$$

This is a non-standard formulation of two separate Neumann problems imposing $\left.\partial_{\boldsymbol{n}_{i}} p_{i}\right|_{\Gamma}=g_{i}$, for $i=1,2$. The extra terms $\gamma h_{E}\left(\partial_{\boldsymbol{n}_{1}} p_{1}-g_{1}, \partial_{\boldsymbol{n}_{1}} q_{1}\right)_{E}$ is unusual for Neumann problems, but it does not compromise the consistency of the method.

\subsubsection{A priori error analysis}

We now adapt the arguments of 14 to analyze numerical properties of the discrete formulation (29). This formulation will then be used in 3.5 to discretize the projection step in presence of an immersed resistive interface.

In the analysis below, we shall make use of the following $(h, \alpha)$-dependent norms, for the coercivity

$$
\|p\|_{h, \alpha}^{2} \stackrel{\text { def }}{=} \sum_{i=1,2}\left\|\nabla p_{i}\right\|_{0, \Omega_{i}}^{2}+\sum_{E \in \mathcal{G}_{h}} \frac{1}{\alpha+\gamma h_{E}}\|\llbracket p \rrbracket\|_{0, E}^{2}
$$


and the continuity

$$
\|p\|_{h, \alpha}^{2} \stackrel{\text { def }}{=}\|p\|_{h, \alpha}^{2}+\sum_{E \in \mathcal{G}_{h}} h_{E}\left\|\partial_{\boldsymbol{n}_{1}} p_{1}\right\|_{0, E}^{2} .
$$

Remark 3.1 Thanks to (7) these norms are equivalent in $N_{h}^{k}$, independently of $h$ and $\alpha$.

The next result shows that, under appropriate regularity assumptions, the discrete formulation (29) is consistent with (24)-(25).

Proposition 3.1 (Galerkin orthogonality) Let $p_{h} \in N_{h}^{k}$ be a solution of (29) and $p \in M$ a solution of (24)-(25). Assume that $p_{i} \in H^{\frac{3}{2}+\epsilon}\left(\Omega_{i}\right)$ for $i=1,2$ and some $\epsilon>0$. Then, there holds

$$
\mathcal{C}_{h, \alpha}\left(p-p_{h}, q_{h}\right)=0,
$$

for all $q_{h} \in N_{h}^{k}$.

Proof. By multiplying (24) ${ }_{1}$ by $q_{h} \in N_{h}^{k}$, integrating over $\Omega_{i}$, summing for $i=1,2$, and using (27), we obtain

$$
\sum_{i=1,2}\left(\nabla p_{i}, \nabla q_{h, i}\right)_{\Omega_{i}}-\sum_{E \in \mathcal{G}_{h}}\left(\partial_{\boldsymbol{n}_{1}} p_{1}, \llbracket q_{h} \rrbracket\right)_{E}=\sum_{i=1,2}\left(f_{i}, q_{h, i}\right)_{\Omega_{i}}
$$

Next, multiplying the boundary condition $(25)$ by $\frac{\alpha}{\alpha+\gamma h_{E}}$ on each interface element $E$ and testing with $q_{h, 1}-q_{h, 2}$, we have

$$
\sum_{E \in \mathcal{G}_{h}} \frac{1}{\alpha+\gamma h_{E}}\left[\alpha\left(\partial_{\boldsymbol{n}_{1}} p_{1}, \llbracket q_{h} \rrbracket\right)_{E}+\left(\llbracket p \rrbracket, \llbracket q_{h} \rrbracket\right)_{E}-\alpha\left(g_{1}, \llbracket q_{h} \rrbracket\right)_{E}\right]=0
$$

At last, multiplying the boundary condition $(25)$ by $-\frac{\alpha \gamma h_{E}}{\alpha+\gamma h_{E}}$ on each interface element $E$ and testing with $\partial_{\boldsymbol{n}_{1}} q_{h, 1}$ *, we get the symmetrization term

$$
\sum_{E \in \mathcal{G}_{h}}-\frac{\gamma h_{E}}{\alpha+\gamma h_{E}}\left[\alpha\left(\partial_{\boldsymbol{n}_{1}} p_{1}, \partial_{\boldsymbol{n}_{1}} q_{h, 1}\right)_{E}+\left(\llbracket p \rrbracket, \partial_{\boldsymbol{n}_{1}} q_{h, 1}\right)_{E}-\alpha\left(g_{1}, \partial_{\boldsymbol{n}_{1}} q_{h, 1}\right)_{E}\right]=0
$$

The sum of (31), (32) and (33) yields

$$
\mathcal{C}_{h, \alpha}\left(p, q_{h}\right)=\mathcal{G}_{h, \alpha}\left(q_{h}\right),
$$

for all $q_{h} \in N_{h}^{k}$, which completes the proof.

The following result provides the coercivity of $\mathcal{C}_{h, \alpha}(\cdot, \cdot)$ with respect to the norm $\|\cdot\|_{h, \alpha}$.

Proposition 3.2 (Coercivity) For $\gamma>0$ and $\theta \in(0,1]$ there holds

$$
\mathcal{C}_{h, \alpha}\left(q_{h}, q_{h}\right) \geq\left(1-\gamma \frac{C_{\mathrm{T}}}{\theta}\right) \sum_{i=1,2}\left\|\nabla q_{h, i}\right\|_{0, \Omega_{i}}^{2}+(1-\theta) \sum_{E \in \mathcal{G}_{h}} \frac{1}{\alpha+\gamma h_{E}}\left\|\llbracket q_{h} \rrbracket\right\|_{0, E}^{2}
$$

${ }^{*}$ Here we consider the side of $\Gamma$ corresponding to $\Omega_{1}$, and test with $\partial_{\boldsymbol{n}_{1}} q_{h, 1}$. However, considering both sides of the interface and testing with any convex combination $\beta \partial_{\boldsymbol{n}_{1}} q_{h, 1}+$ $(\beta-1) \partial_{\boldsymbol{n}_{2}} q_{h, 2}$ would also give a strongly consistent method. 
for all $q_{h} \in N_{h}^{k}$. In particular, for $0<\gamma \leq 1 /\left(4 C_{\mathrm{T}}\right)$ we have

$$
\mathcal{C}_{h, \alpha}\left(q_{h}, q_{h}\right) \geq \frac{1}{2}\left\|q_{h}\right\|_{h, \alpha}^{2}
$$

for all $q_{h} \in N_{h}^{k}$, so that the bilinear form $\mathcal{C}_{h, \alpha}$ is coercive in the norm $\|\cdot\|_{h, \alpha}$ independently of $\alpha$.

Proof. In order to shorten the notation, we drop in this proof the subindex $h$ for the function $q$ belonging to $N_{h}^{k}$. Using Young's inequality, we get

$$
\begin{aligned}
\mathcal{C}_{h, \alpha}(q, q)= & \sum_{i=1,2}\left\|\nabla q_{i}\right\|_{0, \Omega_{i}}^{2}+\sum_{E \in \mathcal{G}_{h}} \frac{1}{\alpha+\gamma h_{E}}\|\llbracket q \rrbracket\|_{0, E}^{2} \\
& -2 \sum_{E \in \mathcal{G}_{h}} \frac{\gamma h_{E}}{\alpha+\gamma h_{E}}\left(\partial_{\boldsymbol{n}_{1}} q_{1}, \llbracket q \rrbracket\right)_{E}-\sum_{E \in \mathcal{G}_{h}} \frac{\alpha \gamma h_{E}}{\alpha+\gamma h_{E}}\left\|\partial_{\boldsymbol{n}_{1}} q_{1}\right\|_{0, E}^{2} \\
\geq & \sum_{i=1,2}\left\|\nabla q_{i}\right\|_{0, \Omega_{i}}^{2}+(1-\theta) \sum_{E \in \mathcal{G}_{h}} \frac{1}{\alpha+\gamma h_{E}}\|\llbracket q \rrbracket\|_{0, E}^{2} \\
& -\gamma \sum_{E \in \mathcal{G}_{h}} \frac{\alpha+\gamma h_{E} / \theta}{\alpha+\gamma h_{E}} h_{E}\left\|\partial_{\boldsymbol{n}_{1}} q_{1}\right\|_{0, E}^{2},
\end{aligned}
$$

for $\theta \in(0,1]$. The last term is controlled using the inverse inequality (7):

$$
\mathcal{C}_{h, \alpha}(q, q) \geq \sum_{i=1,2}\left\|\nabla q_{i}\right\|_{0, \Omega_{i}}^{2}+(1-\theta) \sum_{E \in \mathcal{G}_{h}} \frac{1}{\alpha+\gamma h_{E}}\|\llbracket q \rrbracket\|_{0, E}^{2}-\gamma \frac{C_{T}}{\theta}\left\|\nabla q_{1}\right\|_{0, \Omega_{1}}^{2},
$$

which yields (34).

Proposition 3.3 (Continuity) For $\gamma>0$, there exists a constant $C_{\mathrm{c}}$ depending only on $\gamma$ and $C_{\mathrm{T}}$, such that

$$
\mathcal{C}_{h, \alpha}\left(p_{h}, q\right) \leq C_{\mathrm{c}}\left\|p_{h}\right\|_{h, \alpha}\|q\|_{h, \alpha}
$$

for all $p_{h} \in N_{h}^{k}$ and $q \in M$ with $q_{i} \in H^{\frac{3}{2}+\epsilon}\left(\Omega_{i}\right)$ for $i=1,2$ and some $\epsilon>0$.

Proof. We estimate each term in $\mathcal{C}_{h, \alpha}\left(p_{h}, q\right)$ separately, for $p_{h} \in N_{h}^{k}$ and $q \in M$ with $q_{i} \in H^{\frac{3}{2}+\epsilon}\left(\Omega_{i}\right)$ for $i=1,2$ and $\epsilon>0$. For the first term, we clearly have

$$
\sum_{E \in \mathcal{G}_{h}} \frac{\left\|\llbracket p_{h} \rrbracket\right\|_{0, E}\|\llbracket q \rrbracket\|_{0, E}}{\alpha+\gamma h_{E}} \leq\left\|p_{h}\right\|_{h, \alpha}\|q\|_{h, \alpha} .
$$

Similarly, for the second, there follows

$$
\begin{aligned}
& \sum_{E \in \mathcal{G}_{h}} \frac{\gamma h_{E}}{\alpha+\gamma h_{E}}\left\|\llbracket p_{h} \rrbracket\right\|_{0, E}\left\|\partial_{\boldsymbol{n}_{1}} q_{1}\right\|_{0, E} \leq\left(\sum_{E \in \mathcal{G}_{h}} \frac{\gamma}{\alpha+\gamma h_{E}}\left\|\llbracket p_{h} \rrbracket\right\|_{0, E}^{2}\right)^{1 / 2} \\
&\left(\sum_{E \in \mathcal{G}_{h}} \frac{\gamma h_{E}}{\alpha+\gamma h_{E}} h_{E}\left\|\partial_{\boldsymbol{n}_{1}} q_{1}\right\|_{0, E}^{2}\right)^{1 / 2} \\
& \leq \gamma^{\frac{1}{2}}\left\|p_{h}\right\|_{h, \alpha}\|q\|_{h, \alpha} .
\end{aligned}
$$


The symmetric contribution can be treated similarly, and using (7). Finally, for the last term

$$
\begin{aligned}
& \sum_{E \in \mathcal{G}_{h}} \frac{\alpha \gamma h_{E}}{\alpha+\gamma h_{E}}\left\|\partial_{\boldsymbol{n}_{1}} p_{h, 1}\right\|_{0, E}\left\|\partial_{\boldsymbol{n}_{1}} q_{1}\right\|_{0, E} \leq \gamma\left(\sum_{E \in \mathcal{G}_{h}} \frac{\alpha}{\alpha+\gamma h_{E}} h_{E}\left\|\partial_{\boldsymbol{n}_{1}} p_{h, 1}\right\|_{0, E}^{2}\right)^{1 / 2} \\
&\left(\sum_{E \in \mathcal{G}_{h}} \frac{\alpha}{\alpha+\gamma h_{E}} h_{E}\left\|\partial_{\boldsymbol{n}_{1}} q_{1}\right\|_{0, E}^{2}\right)^{1 / 2} \\
& \leq \gamma C_{\mathrm{T}}^{\frac{1}{2}}\left\|p_{h}\right\|_{h, \alpha}\|q\|_{h, \alpha},
\end{aligned}
$$

where we have used (7). We conclude the proof by collecting all the above estimates.

For each $q \in N$ we define $I_{h}^{k} q \in N_{h}^{k}$ as

$$
\left(I_{h}^{k} q\right)_{\mid \Omega_{i}} \stackrel{\text { def }}{=} I_{h, i}^{k} q_{i}, \text { for } i=1,2,
$$

where $I_{h, i}^{k}$ denotes the standard $L^{2}$-projection onto $M_{h, i}^{k}$. Note that, by construction $I_{h}^{k} q \in N_{h}^{k}$. The following standard approximation result holds.

Lemma 3.1 There exists a constant $C_{\mathrm{I}}>0$ independent of $h$, such that

$$
\left\|q-I_{h}^{k}(q)\right\|_{h, \alpha} \leq C_{\mathrm{I}} h^{k} \sum_{i=1,2}\left\|q_{i}\right\|_{k+1, \Omega_{i}}
$$

for all $q \in N$ with $q_{i} \in H^{k+1}\left(\Omega_{i}\right)$ and $i=1,2$.

The next result is a direct consequence of Propositions 3.1, 3.2 and 3.3 and Lemma 3.1. It provides an optimal error estimate which is uniform in $\alpha$. In particular, it shows that the proposed approach authorizes a vanishing resistance, $r_{\Gamma}=0$, without compromising accuracy.

Corollary 3.1 (Convergence) Let $p \in N$ be the unique solution of (24) -(25) and assume that $p_{i} \in H^{k+1}\left(\Omega_{i}\right)$, for $i=1,2$, and that $0<\gamma \leq 1 /\left(4 C_{T}\right)$. Then (29) has a unique solution $p_{h} \in N_{h}^{k}$ and

$$
\left\|p-p_{h}\right\|_{h, \alpha} \leq C h^{k} \sum_{i=1,2}\left\|p_{i}\right\|_{k+1, \Omega_{i}}
$$

where $C>0$ is a constant independent of $\alpha$ and $h$ (but that depends on $\gamma$ ).

\subsection{Discrete stabilized projection scheme}

With the stabilization à la Nitsche presented in the previous section, we can remove the restriction on the resistance in the projection scheme, and thus consider $r_{\Gamma} \geq 0$, even when the projection step is done with the Poisson formulation. The viscous step (22) is unchanged, but (23) is modified according to the stabilization procedure of (29). The discrete stabilized projection algorithm reads: given an initial velocity $\widetilde{\boldsymbol{u}}^{0} \in \boldsymbol{V}_{h}^{k}$, an initial pressure $p^{0} \in N_{h}^{k}$, solve for 
$n=0, \ldots, N_{T}-1$ :

1. Viscous step: find $\widetilde{\boldsymbol{u}}_{h}^{n+1} \in \boldsymbol{V}_{h}^{k}$ such that

$$
\begin{aligned}
\frac{\rho_{f}}{\delta t}\left(\widetilde{\boldsymbol{u}}_{h}^{n+1}, \boldsymbol{v}_{h}\right)+2 \mu\left(\boldsymbol{\epsilon}\left(\widetilde{\boldsymbol{u}}_{h}^{n+1}\right), \boldsymbol{\epsilon}\left(\boldsymbol{v}_{h}\right)\right)+r_{\Gamma}\left(\widetilde{\boldsymbol{u}}_{h}^{n+1}, \boldsymbol{v}_{h}\right)_{\Gamma}= \\
\left(\boldsymbol{f}^{n+1}, \boldsymbol{v}_{h}\right)+\frac{\rho_{f}}{\delta t}\left(\widetilde{\boldsymbol{u}}_{h}^{n}, \boldsymbol{v}_{h}\right)-\sum_{i=1,2}\left(\boldsymbol{\nabla} p_{h, i}^{n}, \boldsymbol{v}_{h, i}\right)_{\Omega_{i}}+\left(\llbracket p_{h}^{n} \rrbracket, \boldsymbol{v}_{h} \cdot \boldsymbol{n}\right)_{\Gamma},
\end{aligned}
$$

for all $\boldsymbol{v}_{h} \in \boldsymbol{V}_{h}^{k}$.

2. Stabilized projection step $\left(r_{\Gamma} \geq 0\right)$ : find $p_{h}^{n+1} \in N_{h}^{k}$ such that

$$
\begin{aligned}
\mathcal{C}_{h, \alpha}\left(p_{h}^{n+1}, q_{h}\right)= & \frac{\rho_{f}}{\delta t} \sum_{i=1,2}\left(\widetilde{\boldsymbol{u}}_{h, i}^{n+1}, \nabla q_{h, i}\right)_{\Omega_{i}}-\frac{\rho_{f}}{\delta t} \sum_{E \in \mathcal{G}_{h}} \frac{\gamma h_{E}}{\alpha+\gamma h_{E}}\left(\widetilde{\boldsymbol{u}}_{h} \cdot \boldsymbol{n}^{n+1}, \llbracket q_{h} \rrbracket\right)_{E} \\
& -\frac{\rho_{f}}{\delta t} \sum_{E \in \mathcal{G}_{h}} \frac{\alpha \gamma h_{E}}{\alpha+\gamma h_{E}}\left(\widetilde{\boldsymbol{u}}_{h} \cdot \boldsymbol{n}^{n+1}, \partial_{\boldsymbol{n}_{1}} q_{h, 1}\right)_{E},
\end{aligned}
$$

for all $q_{h} \in N_{h}^{k}$, where $\alpha$ is defined in (28) and $\mathcal{C}_{h, \alpha}(\cdot, \cdot)$ in (30).

\subsection{Stability analysis of the projection schemes}

This section is dedicated to the derivation of energy based stability estimates for the two projection schemes. We first discuss in 3.6 .1 the standard Poisson formulation, and then turn in $\$ 3.6 .2$ to the formulation with the Nitsche's interface stabilization terms. We will see that both scheme have a similar energy estimate, except that energy of the stabilized version is still valid for $r_{\Gamma}=0$.

\subsubsection{Non-stabilized projection step}

Let us assume here that $r_{\Gamma}>0$ and consider the formulation (22)-(23), where for the sake of simplicity, we take $\boldsymbol{f}=\mathbf{0}$ and omit the subscripts $h$.

Proposition 3.4 Assume $r_{\Gamma}>0$ and $\boldsymbol{f}=\mathbf{0}$. Let $\left(\widetilde{\boldsymbol{u}}^{n}, p^{n}\right) \in \boldsymbol{V}_{h}^{k} \times N_{h}^{k}$ be the discrete solutions of the projection scheme (22)-(23), for $n=1, \ldots, N_{T}$. Then the scheme (22)-(23) is energy stable, in the sense that

$$
\begin{aligned}
\frac{\rho_{f}}{2}\left\|\widetilde{\boldsymbol{u}}^{N_{T}}\right\|_{0, \Omega}^{2}+\mu \sum_{n=0}^{N_{T}-1} \delta t\left\|\boldsymbol{\epsilon}\left(\widetilde{\boldsymbol{u}}^{n+1}\right)\right\|_{0, \Omega}^{2} & +\frac{r_{\Gamma}}{2} \sum_{n=0}^{N_{T}-1} \delta t\left\|\widetilde{\boldsymbol{u}}^{n+1}\right\|_{0, \Gamma}^{2} \\
+\frac{1}{2 r_{\Gamma}} \sum_{n=0}^{N_{T}-2} \delta t\left\|\llbracket p^{n+1} \rrbracket\right\|_{0, \Gamma}^{2}+\frac{\delta t}{2 \rho_{f}} & \sum_{n=0}^{N_{T}-2} \delta t \sum_{i=1,2}\left\|\nabla p_{i}^{n+1}\right\|_{0, \Omega_{i}}^{2} \\
& \leq \frac{\rho_{f}}{2}\left\|\widetilde{\boldsymbol{u}}^{0}\right\|_{0, \Omega}^{2}+\frac{c_{\Omega} \delta t}{\mu}\left\|p^{0}\right\|_{0, \Omega}^{2},
\end{aligned}
$$

where $c_{\Omega}$ is a constant depending only on the geometry of the domain.

Remark 3.2 The energy estimate (39) provides the standard velocity stability in the discrete $l^{\infty}\left(0, T, L^{2}(\Omega)\right)$ and $l^{2}\left(0, T, H_{0}^{1}(\Omega)\right)$ norms. Moreover, we recover the natural $O(\delta t)$ pressure stabilization provided by the pressure-Poisson 
formulation of the Chorin-Temam projection scheme. Note that due to the presence of the resistive interface $\Gamma$, we obtain an additional $l^{2}\left(0, T, L^{2}(\Gamma)\right)$ control on the pressure jump and $l^{2}\left(0, T, L^{2}(\Gamma)\right)$ on the velocity.

Proof. Testing (22) with $\boldsymbol{v}=\delta t \widetilde{\boldsymbol{u}}^{n+1}$ and using the identity $(a-b, a)=$ $\frac{1}{2}\left(\|a\|^{2}-\|b\|^{2}+\|a-b\|^{2}\right)$, for $n \geq 0$ we get

$$
\begin{array}{r}
\frac{\rho_{f}}{2}\left(\left\|\widetilde{\boldsymbol{u}}^{n+1}\right\|_{0, \Omega}^{2}-\left\|\widetilde{\boldsymbol{u}}^{n}\right\|_{0, \Omega}^{2}+\right. \\
+\delta t r_{\Gamma}\left\|\widetilde{\boldsymbol{u}}^{n+1}\right\|_{0, \Gamma}^{2}=\delta t(\underbrace{\left.\left(\llbracket \widetilde{\boldsymbol{u}}^{n+1}-\widetilde{\boldsymbol{u}}^{n} \|_{0, \Omega}^{2}\right)+2 \mu \delta t \| \boldsymbol{\epsilon}\left(\widetilde{\boldsymbol{u}}^{n+1} \cdot \boldsymbol{n}\right)_{\Gamma}-\sum_{i=1,2}\left(\widetilde{\boldsymbol{u}}_{i}^{n+1}, \boldsymbol{\nabla} p_{i}^{n}\right)_{\Omega_{i}}\right)}_{\mathcal{T}_{1}} .
\end{array}
$$

For $n=0$, the term $\mathcal{T}_{1}$ can be estimated as follows

$$
\mathcal{T}_{1}=\left(\operatorname{div} \widetilde{\boldsymbol{u}}^{1}, p^{0}\right) \leq \frac{\mu}{2}\left\|\boldsymbol{\epsilon}\left(\widetilde{\boldsymbol{u}}^{1}\right)\right\|_{0, \Omega}^{2}+\frac{d C_{\mathrm{K}}^{2}}{2 \mu}\left\|p^{0}\right\|_{0, \Omega}^{2},
$$

where $d$ is the dimension and $C_{\mathrm{K}}$ denotes the constant in Korn's inequality. Hence, with the notation $c_{\Omega}=d C_{\mathrm{K}}^{2} / 2$, inserting this estimate in (40) yields

$$
\frac{\rho_{f}}{2}\left\|\widetilde{\boldsymbol{u}}^{1}\right\|_{0, \Omega}^{2}+\mu \delta t\left\|\boldsymbol{\epsilon}\left(\widetilde{\boldsymbol{u}}^{1}\right)\right\|_{0, \Omega}^{2}+\delta t r_{\Gamma}\left\|\widetilde{\boldsymbol{u}}^{1}\right\|_{0, \Gamma}^{2} \leq \frac{\rho_{f}}{2}\left\|\widetilde{\boldsymbol{u}}^{0}\right\|_{0, \Omega}^{2}+\frac{c_{\Omega} \delta t}{\mu}\left\|p^{0}\right\|_{0, \Omega}^{2} .
$$

For $n \geq 1$, the term $\mathcal{T}_{1}$ requires a different treatment. We first replace $n+1$ by $n$ in (23) and test the resulting expression with $q=\left(\delta t^{2} / \rho_{f}\right) p^{n}$. This yields for $n \geq 1$

$$
\begin{aligned}
\frac{\delta t^{2}}{\rho_{f}} \sum_{i=1,2}\left\|\nabla p_{i}^{n}\right\|_{0, \Omega_{i}}^{2}+\frac{\delta t}{r_{\Gamma}}\left\|\llbracket p^{n} \rrbracket\right\|_{0, \Gamma}^{2} & =-\delta t\left(\operatorname{div} \widetilde{\boldsymbol{u}}^{n}, p^{n}\right)+\delta t\left(\widetilde{\boldsymbol{u}}^{n} \cdot \boldsymbol{n}, \llbracket p^{n} \rrbracket\right)_{\Gamma} \\
& =\delta t \sum_{i=1,2}\left(\widetilde{\boldsymbol{u}}_{i}^{n}, \boldsymbol{\nabla} p_{i}^{n}\right)_{\Omega_{i}} .
\end{aligned}
$$

Therefore, by adding this expression to (40) we get the estimate

$$
\begin{array}{r}
\frac{\rho_{f}}{2}\left(\left\|\widetilde{\boldsymbol{u}}^{n+1}\right\|_{0, \Omega}^{2}-\left\|\widetilde{\boldsymbol{u}}^{n}\right\|_{0, \Omega}^{2}+\left\|\widetilde{\boldsymbol{u}}^{n+1}-\widetilde{\boldsymbol{u}}^{n}\right\|_{0, \Omega}^{2}\right)+2 \mu \delta t\left\|\boldsymbol{\epsilon}\left(\widetilde{\boldsymbol{u}}^{n+1}\right)\right\|_{0, \Omega}^{2} \\
+\delta t r_{\Gamma}\left\|\widetilde{\boldsymbol{u}}^{n+1}\right\|_{0, \Gamma}^{2}+\frac{\delta t^{2}}{\rho_{f}} \sum_{i=1,2}\left\|\boldsymbol{\nabla}_{i}^{n}\right\|_{0, \Omega_{i}}^{2}+\frac{\delta t}{r_{\Gamma}}\left\|\llbracket p^{n} \rrbracket\right\|_{0, \Gamma}^{2} \\
=\delta t(\underbrace{\left(\llbracket p^{n} \rrbracket, \widetilde{\boldsymbol{u}}^{n+1} \cdot \boldsymbol{n}\right)_{\Gamma}+\sum_{i=1,2}\left(\widetilde{\boldsymbol{u}}_{i}^{n}-\widetilde{\boldsymbol{u}}_{i}^{n+1}, \boldsymbol{\nabla} p_{i}^{n}\right)_{\Omega_{i}}}_{\mathcal{T}_{2}})
\end{array}
$$

for $n \geq 1$. By using Young's inequality, we get the following bound for $\mathcal{T}_{2}$

$$
\begin{aligned}
\mathcal{T}_{2} \leq & \frac{1}{2 r_{\Gamma}}\left\|\llbracket p^{n} \rrbracket\right\|_{0, \Gamma}^{2}+\frac{r_{\Gamma}}{2}\left\|\widetilde{\boldsymbol{u}}^{n+1}\right\|_{0, \Gamma}^{2} \\
& +\frac{\rho_{f}}{2 \delta t}\left\|\widetilde{\boldsymbol{u}}^{n+1}-\widetilde{\boldsymbol{u}}^{n}\right\|_{0, \Omega}^{2}+\frac{\delta t}{2 \rho_{f}} \sum_{i=1,2}\left\|\boldsymbol{\nabla} p_{i}^{n}\right\|_{0, \Omega_{i}}^{2} .
\end{aligned}
$$

As a result, the energy estimate (39) follows by inserting (44) into (43), summing over $n=1, \ldots, N_{T}-1$ and adding the first-step energy contribution (41). This completes the proof. $\square$ 


\subsubsection{Stabilized projection step}

The stability of the stabilized projection scheme (37)-38) can be derived in a similar way.

Proposition 3.5 Assume that $r_{\Gamma} \geq 0$ and $\boldsymbol{f}=\mathbf{0}$. Let $\left(\widetilde{\boldsymbol{u}}^{n}, p^{n}\right) \in \boldsymbol{V}_{h}^{k} \times N_{h}^{k}$ be the discrete solutions of the projection scheme with Nitsche's stabilization (37)(38), for $n=1, \ldots, N$. Then the scheme (37)-(38) is stable, in the sense that if $\gamma>0$ is small enough, there exists $C$, independent of $N_{T}, h, \delta t, \mu$ and $r_{\Gamma}$, such that

$$
\begin{aligned}
& \frac{\rho_{f}}{2}\left\|\widetilde{\boldsymbol{u}}^{N_{T}}\right\|_{0, \Omega}^{2}+\mu \sum_{n=0}^{N_{T}-1} \delta t\left\|\boldsymbol{\epsilon}\left(\widetilde{\boldsymbol{u}}^{n+1}\right)\right\|_{0, \Omega}^{2} \\
& +C\left(r_{\Gamma} \sum_{n=0}^{N_{T}-1} \delta t\left\|\widetilde{\boldsymbol{u}}^{n+1}\right\|_{0, \Gamma}^{2}+\sum_{n=0}^{N_{T}-2} \delta t \frac{\left\|\llbracket p^{n+1} \rrbracket\right\|_{0, \Gamma}^{2}}{r_{\Gamma}+\rho_{f} \gamma h / \delta t}+\frac{\delta t}{2 \rho_{f}} \sum_{n=0}^{N_{T}-2} \delta t \sum_{i=1,2}\left\|\nabla p_{i}^{n+1}\right\|_{0, \Omega_{i}}^{2}\right) \\
& \leq \frac{\rho_{f}}{2}\left\|\widetilde{\boldsymbol{u}}^{0}\right\|_{0, \Omega}^{2}+\frac{c_{\Omega} \delta t}{\mu}\left\|p^{0}\right\|_{0, \Omega}^{2},
\end{aligned}
$$

where $c_{\Omega}$ is a constant depending only on the geometry of the domain.

Proof. We first note that for $n=0$ the estimation (41) remains valid. However, for $n \geq 1$, the proof of Proposition 3.4 must be adapted to account for the new terms in (38). To this aim, we replace $n+1$ by $n$ in (38), test the resulting expression with $q=\left(\delta t^{2} / \rho_{f}\right) p^{n}$ and use (34) to obtain, for $n \geq 1$,

$$
\begin{aligned}
&\left(1-\gamma \frac{C_{\mathrm{T}}}{\theta}\right) \frac{\delta t^{2}}{\rho_{f}} \sum_{i=1,2}\left\|\nabla p_{i}^{n}\right\|_{0, \Omega_{i}}^{2}+(1-\theta) \frac{\delta t^{2}}{\rho_{f}} \sum_{E \in \mathcal{G}_{h}} \frac{1}{\alpha+\gamma h_{E}}\left\|\llbracket p^{n} \rrbracket\right\|_{0, E}^{2} \\
& \leq \delta t \sum_{i=1,2}\left(\widetilde{\boldsymbol{u}}_{i}^{n}, \boldsymbol{\nabla} p_{i}^{n}\right)_{\Omega_{i}}-\delta t \sum_{E \in \mathcal{G}_{h}} \frac{\gamma h_{E}}{\alpha+\gamma h_{E}}\left(\widetilde{\boldsymbol{u}}^{n} \cdot \boldsymbol{n}, \llbracket p^{n} \rrbracket\right)_{E} \\
&-\delta t \sum_{E \in \mathcal{G}_{h}} \frac{\alpha \gamma h_{E}}{\alpha+\gamma h_{E}}\left(\widetilde{\boldsymbol{u}}^{n} \cdot \boldsymbol{n}, \partial_{\boldsymbol{n}_{1}} p_{1}^{n}\right)_{E} .
\end{aligned}
$$

By adding this expression to (40), we get for $n \geq 1$

$$
\begin{aligned}
& \frac{\rho_{f}}{2}\left(\left\|\widetilde{\boldsymbol{u}}^{n+1}\right\|_{0, \Omega}^{2}-\left\|\widetilde{\boldsymbol{u}}^{n}\right\|_{0, \Omega}^{2}+\left\|\widetilde{\boldsymbol{u}}^{n+1}-\widetilde{\boldsymbol{u}}^{n}\right\|_{0, \Omega}^{2}\right)+2 \mu \delta t\left\|\boldsymbol{\epsilon}\left(\widetilde{\boldsymbol{u}}^{n+1}\right)\right\|_{0, \Omega}^{2} \\
& +\delta t r_{\Gamma}\left\|\widetilde{\boldsymbol{u}}^{n+1}\right\|_{0, \Gamma}^{2}+\left(1-\gamma \frac{C_{\mathrm{T}}}{\theta}\right) \frac{\delta t^{2}}{\rho_{f}} \sum_{i=1,2}\left\|\nabla p_{i}^{n}\right\|_{0, \Omega_{i}}^{2} \\
& +(1-\theta) \frac{\delta t^{2}}{\rho_{f}} \sum_{E \in \mathcal{G}_{h}} \frac{1}{\alpha+\gamma h_{E}}\left\|\llbracket p^{n} \rrbracket\right\|_{0, E}^{2} \leq \delta t \underbrace{\sum_{i=1,2}\left(\widetilde{\boldsymbol{u}}_{i}^{n}-\widetilde{\boldsymbol{u}}_{i}^{n+1}, \nabla p_{i}^{n}\right)_{\Omega_{i}}}_{\mathcal{T}_{1}} \\
& +\delta t \underbrace{\sum_{E \in \mathcal{G}_{h}}\left(\widetilde{\boldsymbol{u}}^{n+1} \cdot \boldsymbol{n}-\frac{\gamma h_{E}}{\alpha+\gamma h_{E}} \widetilde{\boldsymbol{u}}^{n} \cdot \boldsymbol{n}, \llbracket p^{n} \rrbracket\right)_{E}}_{\mathcal{T}_{2}}-\delta t \underbrace{\sum_{E \in \mathcal{G}_{h}} \frac{\alpha \gamma h_{E}}{\alpha+\gamma h_{E}}\left(\widetilde{\boldsymbol{u}}^{n} \cdot \boldsymbol{n}, \partial_{\boldsymbol{n}_{1}} p_{1}^{n}\right)_{E}}_{\mathcal{T}_{3}} \cdot
\end{aligned}
$$


We now estimate each of the three terms in the right hand-side separately using repeatedly Young's inequality. The term $\mathcal{T}_{1}$ can be estimated as in the proof of Proposition 3.4, that is

$$
\mathcal{T}_{1} \leq \frac{\epsilon_{1}}{2} \frac{\rho_{f}}{\delta t}\left\|\widetilde{\boldsymbol{u}}^{n+1}-\widetilde{\boldsymbol{u}}^{n}\right\|_{0, \Omega}^{2}+\frac{1}{2 \epsilon_{1}} \frac{\delta t}{\rho_{f}} \sum_{i=1,2}\left\|\nabla p_{i}^{n}\right\|_{0, \Omega_{i}}^{2}
$$

The term $\mathcal{T}_{2}$ can be manipulated into

$$
\mathcal{T}_{2}=\underbrace{\sum_{E \in \mathcal{G}_{h}} \frac{\gamma h_{E}}{\alpha+\gamma h_{E}}\left(\left(\widetilde{\boldsymbol{u}}^{n+1}-\widetilde{\boldsymbol{u}}^{n}\right) \cdot \boldsymbol{n}, \llbracket p^{n} \rrbracket\right)_{E}}_{\mathcal{T}_{2,1}}+\underbrace{\sum_{E \in \mathcal{G}_{h}} \frac{\alpha}{\alpha+\gamma h_{E}}\left(\widetilde{\boldsymbol{u}}^{n+1} \cdot \boldsymbol{n}, \llbracket p^{n} \rrbracket\right)_{E}}_{\mathcal{T}_{2,2}},
$$

so that the first term can be estimated, using (7), as follows

$$
\begin{aligned}
\mathcal{T}_{2,1} & \leq \frac{\epsilon_{2}}{2} \frac{\rho_{f}}{\delta t} \sum_{E \in \mathcal{G}_{h}} \frac{\gamma h_{E}}{\alpha+\gamma h_{E}} \gamma h_{E}\left\|\widetilde{\boldsymbol{u}}^{n+1}-\widetilde{\boldsymbol{u}}^{n}\right\|_{0, \Gamma}^{2}+\frac{1}{2 \epsilon_{2}} \frac{\delta t}{\rho_{f}} \sum_{E \in \mathcal{G}_{h}} \frac{1}{\alpha+\gamma h_{E}}\left\|\llbracket p^{n} \rrbracket\right\|_{0, E}^{2} \\
& \leq \frac{\epsilon_{2} \gamma C_{\mathrm{T}}}{2} \frac{\rho_{f}}{\delta t}\left\|\widetilde{\boldsymbol{u}}^{n+1}-\widetilde{\boldsymbol{u}}^{n}\right\|_{0, \Omega}^{2}+\frac{1}{2 \epsilon_{2}} \frac{\delta t}{\rho_{f}} \sum_{E \in \mathcal{G}_{h}} \frac{1}{\alpha+\gamma h_{E}}\left\|\llbracket p^{n} \rrbracket\right\|_{0, E}^{2},
\end{aligned}
$$

and similarly, the second can be controlled as

$$
\begin{aligned}
\mathcal{T}_{2,2} \leq & \frac{\epsilon_{3}}{2} \frac{\alpha \rho_{f}}{\delta t} \sum_{E} \frac{\alpha}{\alpha+\gamma h_{E}}\left\|\widetilde{\boldsymbol{u}}^{n+1}\right\|_{0, E}^{2}+\frac{1}{2 \epsilon_{3}} \frac{\delta t}{\rho_{f}} \sum_{E \in \mathcal{G}_{h}} \frac{1}{\alpha+\gamma h_{E}}\left\|\llbracket p^{n} \rrbracket\right\|_{0, E}^{2} \\
& \leq \frac{\epsilon_{3}}{2} r_{\Gamma}\left\|\widetilde{\boldsymbol{u}}^{n+1}\right\|_{0, \Gamma}^{2}+\frac{1}{2 \epsilon_{3}} \frac{\delta t}{\rho_{f}} \sum_{E \in \mathcal{G}_{h}} \frac{1}{\alpha+\gamma h_{E}}\left\|\llbracket p^{n} \rrbracket\right\|_{0, E}^{2} \cdot
\end{aligned}
$$

Finally, for the last term in (46) we consider the decomposition

$$
\begin{aligned}
\mathcal{T}_{3}= & \underbrace{\sum_{E \in \mathcal{G}_{h}} \frac{\alpha \gamma h_{E}}{\alpha+\gamma h_{E}}\left(\widetilde{\boldsymbol{u}}^{n} \cdot \boldsymbol{n}-\widetilde{\boldsymbol{u}}^{n+1} \cdot \boldsymbol{n}, \partial_{\boldsymbol{n}_{1}} p_{1}^{n}\right)_{E}}_{\mathcal{T}_{3,1}} \\
& +\underbrace{\sum_{E \in \mathcal{G}_{h}} \frac{\alpha \gamma h_{E}}{\alpha+\gamma h_{E}}\left(\widetilde{\boldsymbol{u}}^{n+1} \cdot \boldsymbol{n}, \partial_{\boldsymbol{n}_{1}} p_{1}^{n}\right)_{E}}_{\mathcal{T}_{3,2}}
\end{aligned}
$$

in which the first term can be bounded, using (7), as

$$
\begin{aligned}
\mathcal{T}_{3,1} \leq & \sum_{E \in \mathcal{G}_{h}}\left(\frac{\alpha}{\alpha+\gamma h_{E}}\right)\left(\gamma h_{E}\right)^{\frac{1}{2}}\left\|\widetilde{\boldsymbol{u}}^{n+1}-\widetilde{\boldsymbol{u}}^{n}\right\|_{0, E}\left(\gamma h_{E}\right)^{\frac{1}{2}}\left\|\partial_{\boldsymbol{n}_{1}} p_{1}^{n}\right\|_{0, E} \\
& \leq \frac{\epsilon_{4} \gamma C_{\mathrm{T}}}{2} \frac{\rho_{f}}{\delta t}\left\|\widetilde{\boldsymbol{u}}^{n+1}-\widetilde{\boldsymbol{u}}^{n}\right\|_{0, \Omega}^{2}+\frac{\gamma C_{\mathrm{T}}}{2 \epsilon_{4}} \frac{\delta t}{\rho_{f}} \sum_{i=1,2}\left\|\nabla p_{i}^{n}\right\|_{0, \Omega_{i}}^{2}
\end{aligned}
$$

and the second, similarly, as

$$
\begin{aligned}
\mathcal{T}_{3,2} & \leq \sum_{E \in \mathcal{G}_{h}}\left(\frac{\alpha}{\alpha+\gamma h_{E}}\right)^{\frac{1}{2}} \alpha^{\frac{1}{2}}\left\|\widetilde{\boldsymbol{u}}^{n+1}\right\|_{0, E}\left(\frac{\gamma h_{E}}{\alpha+\gamma h_{E}}\right)^{\frac{1}{2}}\left(\gamma h_{E}\right)^{\frac{1}{2}}\left\|\partial_{\boldsymbol{n}_{1}} p_{1}^{n}\right\|_{0, E} \\
& \leq \frac{\epsilon_{5}}{2} r_{\Gamma}\left\|\widetilde{\boldsymbol{u}}^{n+1}\right\|_{0, \Gamma}^{2}+\frac{\gamma C_{\mathrm{T}}}{2 \epsilon_{5}} \frac{\delta t}{\rho_{f}} \sum_{i=1,2}\left\|\nabla p_{i}^{n}\right\|_{0, \Omega_{i}}^{2} .
\end{aligned}
$$


Therefore, by collecting the above estimations into (46), for $n \geq 1$ we get

$$
\begin{gathered}
\frac{\rho_{f}}{2}\left\|\widetilde{\boldsymbol{u}}^{n+1}\right\|_{0, \Omega}^{2}+2 \mu \delta t\left\|\boldsymbol{\epsilon}\left(\widetilde{\boldsymbol{u}}^{n+1}\right)\right\|_{0, \Omega}^{2}+\left[1-\epsilon_{1}-\gamma C_{\mathrm{T}}\left(\epsilon_{2}+\epsilon_{4}\right)\right] \frac{\rho_{f}}{2}\left\|\widetilde{\boldsymbol{u}}^{n+1}-\widetilde{\boldsymbol{u}}^{n}\right\|_{0, \Omega}^{2} \\
+\left(1-\frac{\epsilon_{3}}{2}-\frac{\epsilon_{5}}{2}\right) r_{\Gamma} \delta t\left\|\widetilde{\boldsymbol{u}}^{n+1}\right\|_{0, \Gamma}^{2} \\
+\left[1-\gamma \frac{C_{\mathrm{T}}}{\theta}-\frac{1}{2 \epsilon_{1}}-\gamma C_{\mathrm{T}}\left(\frac{1}{2 \epsilon_{4}}+\frac{1}{2 \epsilon_{5}}\right)\right] \frac{\delta t^{2}}{\rho_{f}} \sum_{i=1,2}\left\|\nabla p_{i}^{n}\right\|_{0, \Omega_{i}}^{2} \\
+\left(1-\theta-\frac{1}{2 \epsilon_{2}}-\frac{1}{2 \epsilon_{3}}\right) \frac{\delta t^{2}}{\rho_{f}} \sum_{E \in \mathcal{G}_{h}} \frac{1}{\alpha+\gamma h_{E}}\left\|\llbracket p^{n} \rrbracket\right\|_{0, E}^{2} \leq \frac{\rho_{f}}{2}\left\|\widetilde{\boldsymbol{u}}^{n}\right\|_{0, \Omega}^{2} . \quad(47)
\end{gathered}
$$

We now choose $\oplus^{\dagger}$ the constants $\theta, \epsilon_{i}(i=1, \ldots, 5)$ and $\gamma$ small enough (depending on $C_{\mathrm{T}}$ ) so that the corresponding terms in the left hand side of (47) remain positive. Then we sum over $n=1, \ldots, N_{T}-1$, and add the first-step contribution (41) to obtain (45), which completes the proof.

Remark 3.3 It is worth noticing that the energy estimate (45) is obtained thanks to an appropriate balance between the numerical dissipation $\frac{\rho_{f}}{2}\left\|\widetilde{\boldsymbol{u}}^{n+1}-\widetilde{\boldsymbol{u}}^{n}\right\|_{0, \Omega}^{2}$, provided by the viscous step (37), and the pressure control on $\left\|p^{n}\right\|_{h, \alpha}^{2}$ given by the pressure-Poisson equation (38).

\section{$4 \quad$ Numerical tests}

This section is devoted to the numerical validation of the proposed formulations. First, in 4.1. we design a simple test to investigate the convergence property of the Nitsche interface Poisson problem (29). Next, we benchmark the fractional step approach on a "quasi-Poiseuille flow" in a two-dimensional straight tube in $\$ 4.2$ and on a model of a stented Abdominal Aortic Aneurysm (AAA) in \$4.3.

\subsection{Poisson problem with a resistive interface}

We assess in this section the convergence rate of (29) on a simple analytical solution. We investigate in particular the independence of the convergence coefficient with respect to $\alpha$, see Corollary 3.1

To this purpose, on a rectangular domain $\Omega=[-1,1] \times[0,1]$, we consider the resistive interface $\Gamma=\{0\} \times[0,1]$ and the function

$$
p_{\alpha}=\left\{\begin{array}{ll}
\frac{\alpha^{2}}{2\left(1+\alpha^{2}\right)}(A+B y+C \sin 3 \pi y)+\frac{D x}{1+\alpha^{2}} \sin 3 \pi y & x<0 \\
-\frac{\alpha^{2}}{2\left(1+\alpha^{2}\right)}(A+B y+C \sin 3 \pi y)+\frac{D x}{1+\alpha^{2}} \sin 3 \pi y & x>0
\end{array},\right.
$$

with given constants $A=3, B=10, C=2, D=4$. This function $p_{\alpha}$ is chosen an analytical solution of problem (24)-(25), with adequate non-homogeneous Dirichlet boundary conditions. Note that in $\Omega_{i}, i=1,2$, the $L^{2}, H^{1}$ and $H^{2}$

\footnotetext{
$\dagger^{\dagger}$ A possible parameter configuration is $\theta=\frac{1}{8}, \epsilon_{1}=\frac{3}{4}, \epsilon_{2}=3, \epsilon_{3}=\frac{3}{4}, \epsilon_{4}=\frac{1}{8}, \epsilon_{5}=\frac{1}{2}$, in combination with $\gamma C_{\mathrm{T}}<\frac{1}{40}$.
} 
norms of the function $p_{\alpha}$ are bounded independently of $\alpha$. The source term $f$ and the interface source term $g_{1}\left(=-g_{2}\right)$ in (24) $-(25)$ are defined by

$$
f(x, y)=-\Delta p_{\alpha}, \quad g_{1}(y)=\frac{D \sin 3 \pi y+\alpha(A+B y+C \sin 3 \pi y)}{1+\alpha^{2}}
$$

for any value of $\alpha \geq 0$. The numerical solution of the stabilized formulation (29) with the sources $f$ and $g_{1}, g_{2}$ on a triangular mesh of size $h$ is denoted by $p_{\alpha, h}$. It is plotted in Figure 2. Note that when $\alpha>0$ the pressure is discontinuous, as it is generally the case for the interface model.
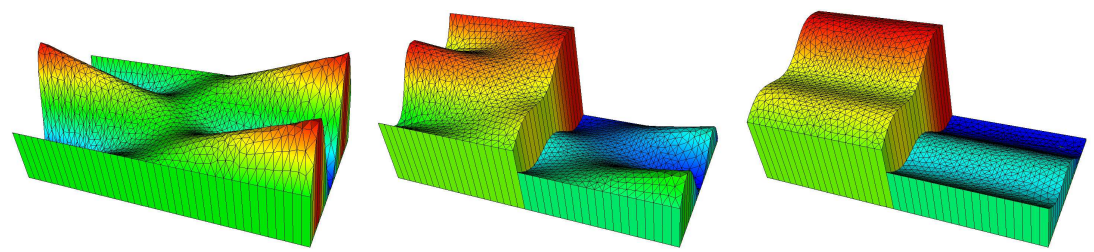

Figure 2: Numerical solutions of the pressure problem. From left to right: $\alpha=0.001,1,100$. (for layout purposes, the selected mesh is quite coarse: $h=$ $5 E-3)$.

Results are summarized in Figure 3, showing in a double logarithmic scale the relative error in $H^{1}$-seminorm, defined by

$$
e_{1, \Omega}=\sum_{i=1,2} \frac{\left\|\nabla\left(p_{\alpha}-p_{\alpha, h}\right)\right\|_{0, \Omega_{i}}}{\left\|\nabla p_{\alpha}\right\|_{0, \Omega_{i}}}
$$

for different discretization sizes. We clearly obtain linear convergence rates and errors which are essentially independent of $\alpha$, as expected (Corollary 3.1).

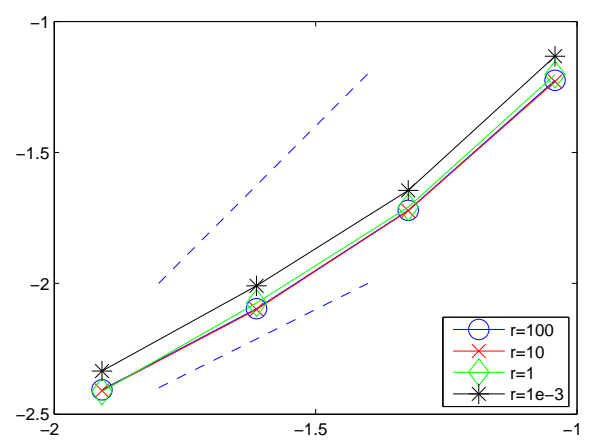

Figure 3: Relative error ( $H^{1}$-seminorm) in double logarithmic scale, for different values of resistance $(\alpha=0.001,1,10,100)$. Mesh sizes $h=10^{-2}, 5 \cdot 10^{-3}, 2.5$. $10^{-3}, 1.25 \cdot 10^{-3}$. The curves are almost superimposed, the topmost curve corresponds to $\alpha=0.001$. Dashed lines show the reference curves for first and second order accuracy. 


\subsection{Quasi-Poiseuille Flow}

The purpose of this numerical test is to compare the results given by the monolithic approach (i.e. the direct discretization in time of (1) by implicit Euler and stabilized $P_{1} / P_{1}$ finite element spaces [10]) and the projection methods (22)-(23) or (37)-(38), in a case when a stationary approximate solution is known. The principle is the following: we consider a non stationary flow in a straight $2 \mathrm{D}$ channel of length $L$ and width $2 b$, with a porous interface $\Gamma=\left\{\frac{L}{2}\right\} \times(-b, b)$ located at the middle of the domain (Figure 4, left). We impose a normal stress $P_{\text {in }}$ at the inlet and $P_{\text {out }}$ at the outlet. On the top and bottom boundaries, a no-slip condition $\boldsymbol{u}=\mathbf{0}$ is imposed. The numerical simulation starts from 0 to reach after a certain time the stationary state, whose approximation can be computed as follows.
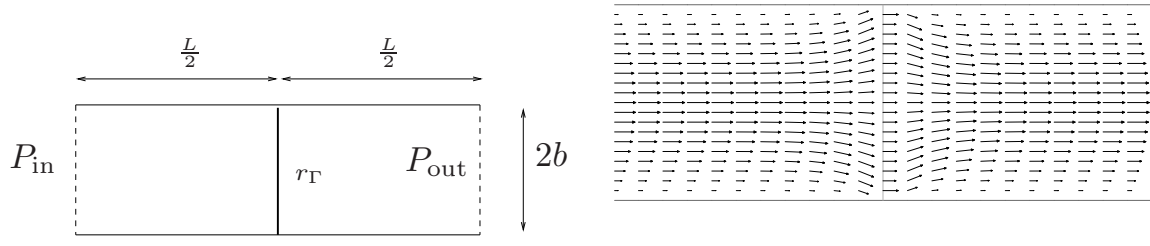

Figure 4: The 2D tube benchmark: straight channel with a straight porous interface. Left: sketch of the domain and boundary conditions. Right: zoom of a numerical solution around the interface $\left(r_{\Gamma}=100\right)$. For the sake of clarity, we show a relatively coarse mesh.

Let $r_{\Gamma}$ be the resistance of the interface. In a first approximation we assume that, for any $r_{\Gamma} \geq 0$, a standard Poiseuille flow is established in both subdomain用. Under this assumption, introducing the flow resistance $R_{2 D}=\frac{3 \mu L}{2 b^{2}}$ of the subdomains, and denoting with $P_{i}$ the pressures at the side $i$ of the interface, we obtain the following relations for the mean velocity

$$
\bar{u}=\frac{P_{1}-P_{\text {in }}}{R_{2 D}}=\frac{1}{r_{\Gamma}}\left(P_{1}-P_{2}\right)=\frac{P_{2}-P_{\text {out }}}{R_{2 D}} .
$$

This yields approximate solutions for the mean velocity and for the resulting pressure jump

$$
\bar{u} \approx \frac{P_{\text {in }}-P_{\text {out }}}{R_{2 D}+r_{\Gamma}+R_{2 D}}, \quad P_{1}-P_{2} \approx\left(P_{\text {in }}-P_{\text {out }}\right) \frac{r_{\Gamma}}{R_{2 D}+r_{\Gamma}+R_{2 D}} .
$$

We use the parameters $L=4 \mathrm{~cm}, b=0.2 \mathrm{~cm}$, the viscosity $\mu=0.04 \frac{\mathrm{g}}{\mathrm{cms}}$, a resistance $r_{\Gamma}=100 \frac{\mathrm{g}}{\mathrm{cm}^{2} \mathrm{~s}}$, a constant pressure drop of $1000 \frac{\mathrm{g}}{\mathrm{cm} \mathrm{s}^{2}}$, resulting in the following values for the flow rate and the pressure jump across $\Gamma$ :

$$
\Phi_{\Gamma} \approx 3.226 \frac{\mathrm{cm}^{3}}{\mathrm{~s}}, \quad \llbracket P \rrbracket \approx 806.5 \frac{\mathrm{g}}{\mathrm{cm} \mathrm{s}^{2}} .
$$

\footnotetext{
${ }^{\ddagger}$ Note that the Poiseuille flow is not a solution to the interface problem (1): actually, there is a boundary layer around the interface, allowing to pass from the Poiseuille parabolic profile to a quasi-flat profile on the interface (Figure 4 right).
} 

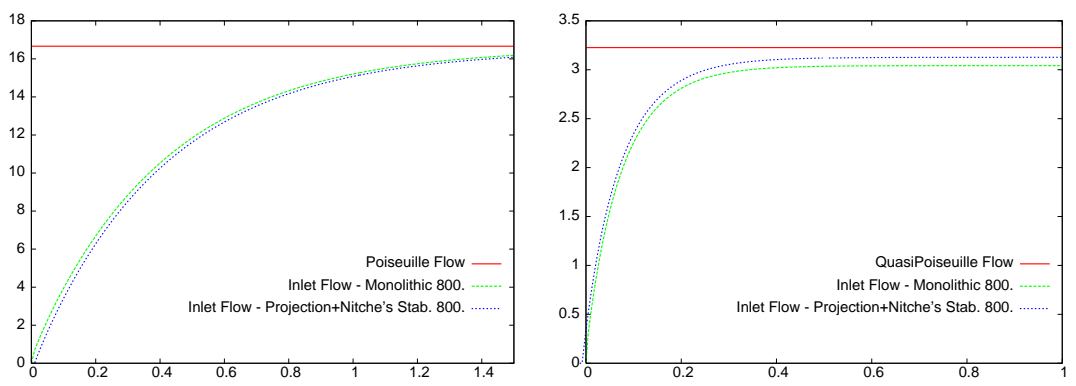

Figure 5: Flow as a function of time. Comparison between monolithic [10], projection and stabilized projection (37)-(38) methods. FE grid of 800 elements. Left: $r_{\Gamma}=0$, right $r_{\Gamma}=100$.
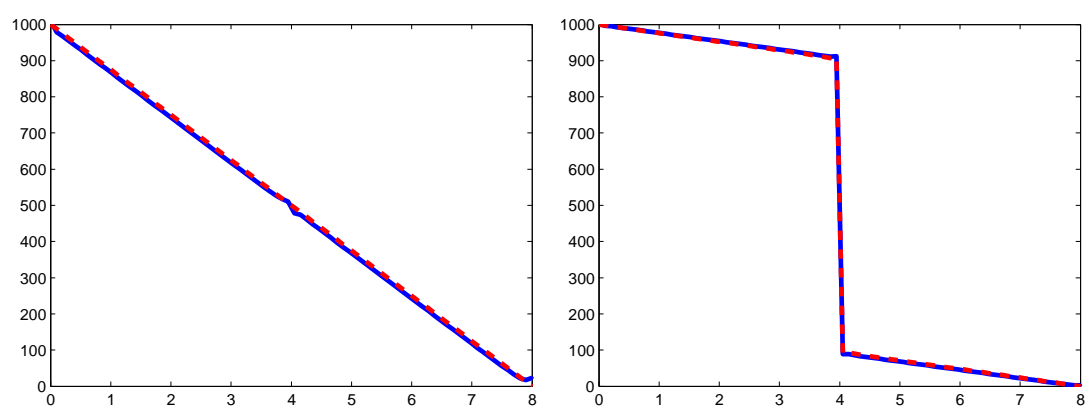

Figure 6: Final pressure as a function of space. Comparisons of the pressure profiles along the middle line of the domain, obtained with monolithic formulation (solid line, cf. [10]) and with the projection scheme (dashed line). Curves are almost superimposed. Grid size: 800 elements, $\delta t=5 \times 10^{-3}$; left: $r_{\Gamma}=0$, right: $r_{\Gamma}=100$.

To compare the monolithic and the projection schemes, in Figure 5 we have reported the time course of the flow rate $\Phi_{\Gamma}(t)$ through the porous interface, for $r_{\Gamma}=0$ and $r_{\Gamma}=100$. The corresponding pressure profiles at final time are depicted in Figure 6. We can observe that both formulations, monolithic and projection based, give similar results. Finally, let us mention that the numerical experiments showed that the stabilized method (37)-(38) and the non-stabilized (22)-(23) provide almost identical results for $r_{\Gamma}=100$. We omit this comparison for the sake of conciseness.

\subsection{Stented aneurysm}

We conclude presenting an application of the resistive immersed interface model to a stented Abdominal Aortic Aneurysm (AAA).

Our model geometry (shown with its finite element mesh in Figure 7), represents a segment of a cylindrical vessel of length $L=16 \mathrm{~cm}$ and diameter $d_{0}=1.7 \mathrm{~cm}$, whose central section contains an aneurysm of length $L_{\text {anev }}=4 \mathrm{~cm}$, of maximum diameter $d_{\mathrm{anev}}=3.4 \mathrm{~cm}$. The stent is modeled as a cylindrical 

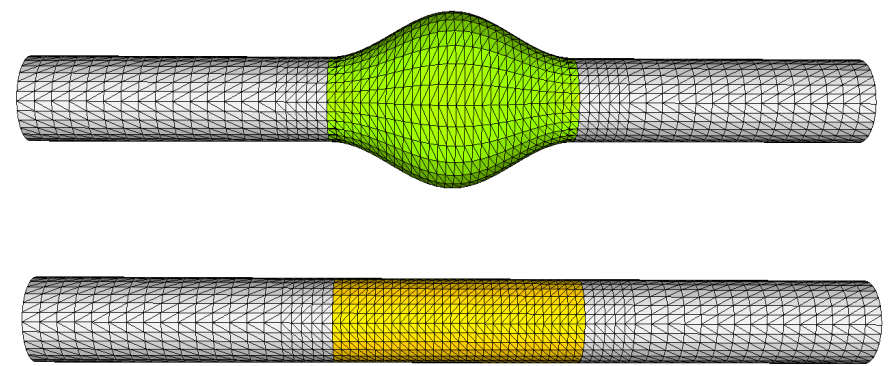

Figure 7: Model geometry of a stented Abdominal Aortic Aneurysm. On the top, the outer boundary composed of vessel wall and aneurysm wall. At the bottom, the inner mesh of the porous interface defining the stent.

porous interface $\Gamma$, with a diameter equal to the vessel diameter $d_{0}$ and length equal to the aneurysm length $L_{\text {anev }}$.

\subsubsection{Mesh considerations}

The aneurysm surface has been designed analytically, according to a dilatation of an initial cylindrical vessel (Figure [7, top). Accordingly, the stent surface has been defined as the boundary of the undeformed cylinder (Figure 7 bottom). The volume mesh has been generated using GHS3D $\$$.

We used elements of the same order for velocity and pressure (P1/P1), continuous on the subdomains separated by the stent. In order to allow for the pressure approximation to jump across the porous stent, the nodes on the interface were doubled (i.e. the mesh has been cracked on $\Gamma$ ).

\subsubsection{Results}

We performed numerical simulations imposing an inlet parabolic profile with a
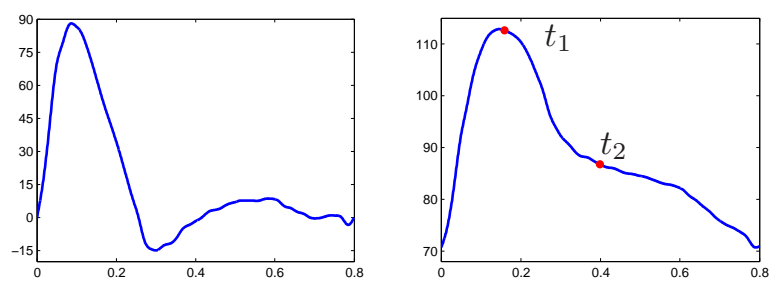

Figure 8: Left: inlet flow rate (in $\mathrm{cm}^{3} \mathrm{~s}^{-1}$ ). Right: outlet pressure profile (in $\mathrm{mmHg}$ ), obtained with $R_{\text {out }}=7 \cdot 10^{3} \frac{\mathrm{g}}{\mathrm{scm}^{4}}, C_{\text {out }}=1.43 \cdot 10^{-5} \frac{\mathrm{g} \mathrm{s}^{2}}{\mathrm{~cm}^{4}}$.

given flow rate (Figure 8 left), and using a Windkessel RC model at the outlet,

\footnotetext{
$\S$ http://www-roc.inria.fr/gamma/gamma/ghs3d/ghs.php
} 
Table 1: GMRES iteration for projection step: Nitsche stabilization $(\gamma=0.08)$ vs. non-stabilized problem $(\gamma=0)$. Values are re-normalized with respect to the minimum.

\begin{tabular}{lcc}
\hline \hline$r_{\Gamma}$ & \# iterations $(\gamma=0.08)$ & \# iterations $(\gamma=0)$ \\
\hline 1 & 1 & 1.89 \\
$10^{-2}$ & 1.07 & 3.68 \\
$10^{-4}$ & 1.07 & 4.28 \\
$10^{-6}$ & 1.07 & 5.02 \\
$10^{-8}$ & 1.07 & 8.55 \\
$10^{-12}$ & 1.07 & $>30$ \\
0 & 1.07 & - \\
\hline
\end{tabular}

calculating the outlet pressure according to

$$
\frac{d p_{\text {out }}}{d t}=\frac{1}{R_{\text {out }} C_{\text {out }}}\left(R_{\text {out }} \Phi-p_{\text {out }}\right) .
$$

The parameter $R_{\text {out }}$ and $C_{\text {out }}$ have been determined in order to obtain a physiological pressure pulse (Figure 8, right).

Table 1 highlights the effect of the interface stabilization, showing, for different values of $r_{\Gamma}$ (tending to zero), the needed GMRES iteration to solve the linear systems arising from the projection step. Without the Nitsche interface penalization, the system (22)-(23) becomes ill conditioned, while the stabilized formulation (37)-(38) is characterized by a condition number independent from $r_{\Gamma}$. This confirms the numerical interest of the proposed approach.

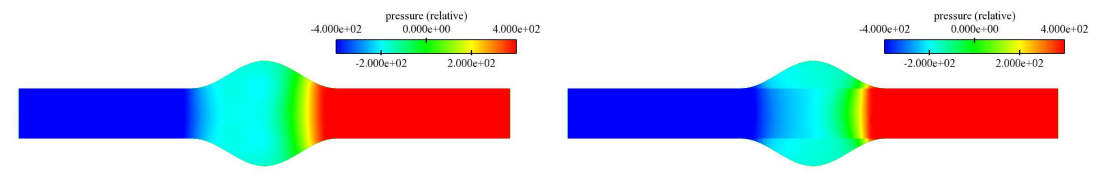

Figure 9: Pressure contour (respect to zero average pressure) at the maximum of the pressure pulse (systole) obtained the fractional step approach, $r_{\Gamma}=0$ (left) and $r_{\Gamma}=20$ (right). The pressure ranges between -0.31 and $0.31 \mathrm{mmHg}$ (corresponding to -400 and $400 \frac{\mathrm{g}}{\mathrm{cm} \cdot \mathrm{s}^{2}}$. .

For illustration purposes, we give some results obtained with the fractional step approach for $r_{\Gamma}=0$ (no stent) and $r_{\Gamma}=20$. Figure 9 shows the pressure contours on a axial cross sectional plane. The continuity of the pressure in clearly observed for $r_{\Gamma}=0$, while for $r_{\Gamma}=20$ we notice a relatively small pressure jump across the stent. In fact, in this particular geometry the stent surface is parallel to the main flow direction and the normal velocity remains small. The effect of the porous interface is better visible looking at the normal derivative of the velocity along the stent axis, as shown Figure 10.

Finally, Figures 11 and 12 show the velocity fields along an axial plane. The main effects are a drastic velocity reduction during the systole $\left(t_{1}\right)$, and a limited recirculation within the aneurysm in the second phase of the cycle. 

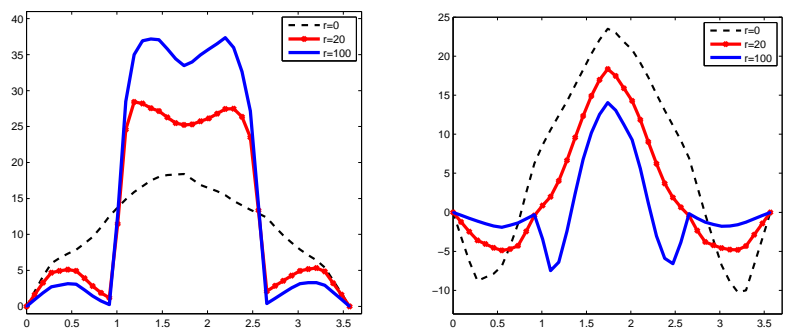

Figure 10: Axial velocity component along a radial direction. Comparison of the results for $r_{\Gamma}=0,20,100$. Left: peak of the pressure profile, right: end of the systolic phase (instants $t_{1}$ and $t_{2}$ in Figure 8).

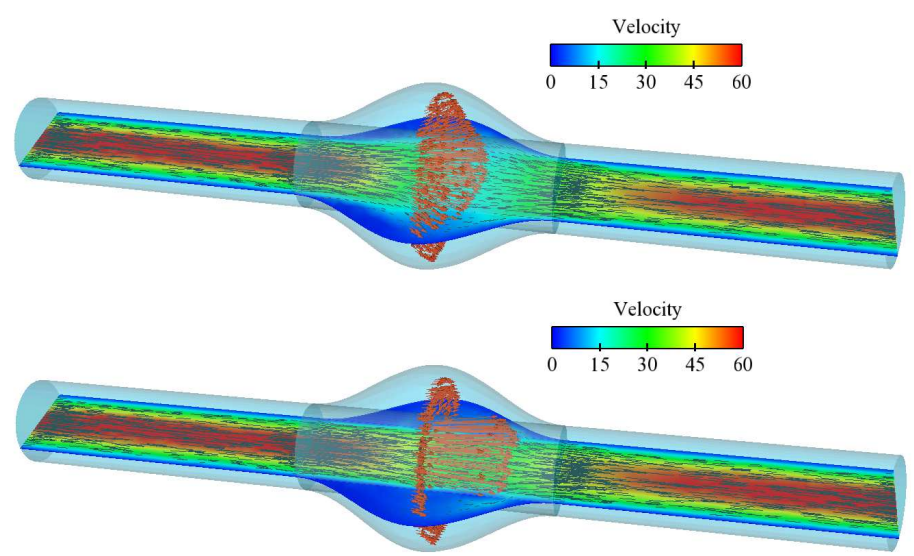

Figure 11: Projection scheme for porous stent. Velocity field at the instants of the cycle indicated by $t_{1}$ (in Figure 8), for $r_{\Gamma}=0$ (top) and $r_{\Gamma}=20$ (bottom).

\section{Conclusions}

In this paper, we presented and analyzed a projection method for the numerical simulation of an incompressible fluid through a porous interface, starting from the monolithic formulation introduced in [10. The discrete fractional step scheme has been derived by introducing an appropriate splitting of the interface resistance conditions. In addition, we showed that a suitable Nitsche interface treatment of the resulting pressure conditions yields a robust method for any value of the interface resistance. This is particularly appealing for situations (stents with holes and heart valve simulations, for instance) in which the resistance parameter takes extreme values (as 0 or $+\infty$ ). The convergence properties of the obtained modified projection step and the stability in time of the different formulations are analyzed. These theoretical results have then been illustrated via numerical experiments, performed on simple benchmarks and on a three dimensional model of aortic aneurysm. 

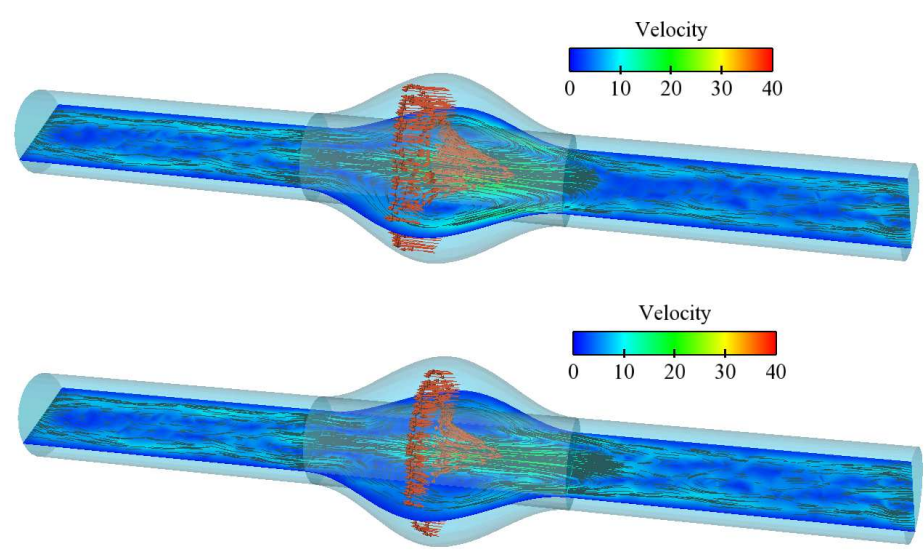

Figure 12: Projection scheme for porous stent. Velocity field at the instants of the cycle indicated by $t_{2}$ (in Figure 8), for $r_{\Gamma}=0$ (top) and $r_{\Gamma}=20$ (bottom).

\section{References}

[1] G. Allaire. Homogenization of the Navier-Stokes equations in open sets perforated with tiny holes. II. Noncritical sizes of the holes for a volume distribution and a surface distribution of holes. Arch. Rational Mech. Anal., 113(3):261-298, 1991.

[2] M. Astorino, S. Shadden, and J-F. Gerbeau. A robust and efficient valve model. submitted, 2010.

[3] Matteo Astorino, Franz Chouly, and Miguel A. Fernández. Robin based semi-implicit coupling in fluid-structure interaction: Stability analysis and numerics. SIAM Journal on Scientific Computing, 31(6):4041-4065, 2009.

[4] Badia, S. and Codina, R. Convergence analysis of the FEM approximation of the first order projection method for incompressible flows with and without the inf-sup condition. Numerische Mathematik, 107:533-557, 2007.

[5] Becker, R. and Hansbo, P. and Stenberg, R. A finite element method for domain decomposition with non-matching grids. Mathematical Modeling and Numerical Analysis, 37(2):209-225, 2003.

[6] S.C. Brenner and L.R. Scott. The mathematical theory of finite element methods. Springer Verlag, 2002.

[7] Chorin, A.J. Numerical solution of the Navier-Stokes equations. Mathematics of Computation, 22:745-762, 1968.

[8] P.G. Ciarlet. The finite element method for elliptic problems, volume 40 of Classics in Applied Mathematics. Society for Industrial and Applied Mathematics (SIAM), Philadelphia, PA, 2002. Reprint of the 1978 original [North-Holland, Amsterdam; MR0520174 (58 \#25001)]. 
[9] Fernández, M.A. and Gerbeau, J.-F. and Grandmont, C. A projection semi-implicit scheme for the coupling of an elastic structure with an incompressible fluid. Int. J. Numer. Meth. Engrg., 69(4):794-821, 2007.

[10] Fernández, M.A. and Gerbeau, J.-F. and Martin, V. Numerical simulation of blood flows through a porous interface. Mathematical Modeling and Numerical Analysis, 42(6):961-990, 2008.

[11] K. Goda. A multistep technique with implicit difference schemes for calculating two- or three-dimensional cavity flows. Journal of Computational Physics, 30:76-95, 1979.

[12] J.L Guermond, P. Minev, and J. Shen. An overview of projection methods for incompressible flows. Comp. Meth. Appl. Mech. Engrg., 195(4447):6011-6045, 2006.

[13] Peter Hansbo. Nitsche's method for interface problems in computational mechanics. GAMM-Mitt., 28(2):183-206, 2005.

[14] Juntunen, M. and Stenberg, R. Nitsche's Method for General Boundary Conditions. Mathematics of Computation, 78(267):1353-1374, 2009.

[15] J. Nitsche. über ein Variationsprinzip zur Lösung von Dirichlet-Problemen bei Verwendung von Teilräumen, die keinen Randbedingungen unterworfen sind. Abh. Math. Sem. Univ. Hamburg, 36:9-15, 1971.

[16] A. Quaini and A. Quarteroni. A semi-implicit approach for fluid-structure interaction based on an algebraic fractional step method. Math. Models Methods Appl. Sci., 17(6):957-983, 2007.

[17] Temam, R. Sur l'approximation de la solution des equations de NavierStokes par la méthode des pas fractionaires I. Archive for Rational Mechanics and Analysis, 32:135-153, 1969.

[18] V. Thomée. Galerkin finite element methods for parabolic problems, volume 25 of Springer Series in Computational Mathematics. Springer-Verlag, Berlin, second edition, 2006.

[19] LJP Timmermans, PD Minev, and FN Van De Vosse. An approximate projection scheme for incompressible flow using spectral elements. International Journal for Numerical Methods in Fluids, 22(7):673-688, 1996. 


\section{Contents}

\begin{tabular}{lll}
\hline & Introduction & 3
\end{tabular}

2 Incompressible fluid through a porous interface 4

2.1 Preliminaries ...................... 5

\begin{tabular}{|lll}
\hline & Projection method for porous interface problems & 6
\end{tabular}

3.1 A domain-decomposition point of view . . . . . . . . . . 7

3.2 Fractional step for the immersed porous interface model . . . . . 8

3.3 Discrete projection scheme . . . . . . . . . . . . . 9

3.4 Stabilized projection via Nitsche interface method . . . . . . . . 9

3.4.1 Nitsche interface method with a resistive interface . . . . 10

3.4.2 A priori error analvsis . . . . . . . . . . . . . . 11

3.5 Discrete stabilized projection scheme . . . . . . . . . . . . . . 14

3.6 Stability analysis of the projection schemes . . . . . . . . . . . 15

3.6.1 Non-stabilized projection step . . . . . . . . . . . . . . 15

3.6.2 Stabilized projection step . . . . . . . . . . . . . 17

$\begin{array}{lll}4 & \text { Numerical tests } & 19\end{array}$

4.1 Poisson problem with a resistive interface . . . . . . . . . . . . . 19

4.2 Quasi-Poiseuille Flow . . . . . . . . . . . . . . . . . . . . . . . . . . . . . . 21

4.3 Stented aneurysm . . . . . . . . . . . . . . . . . . . . 22

4.3 .1 Mesh considerations . . . . . . . . . . . . . . 23

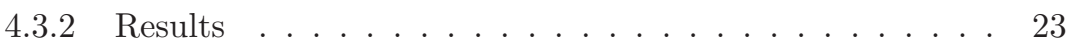

\begin{tabular}{|ll|}
5 & Conclusions \\
\hline
\end{tabular} 
Unité de recherche INRIA Rocquencourt Domaine de Voluceau - Rocquencourt - BP 105 - 78153 Le Chesnay Cedex (France)

Unité de recherche INRIA Futurs : Parc Club Orsay Université - ZAC des Vignes 4, rue Jacques Monod - 91893 ORSAY Cedex (France)

Unité de recherche INRIA Lorraine : LORIA, Technopôle de Nancy-Brabois - Campus scientifique 615, rue du Jardin Botanique - BP 101 - 54602 Villers-lès-Nancy Cedex (France)

Unité de recherche INRIA Rennes : IRISA, Campus universitaire de Beaulieu - 35042 Rennes Cedex (France)

Unité de recherche INRIA Rhône-Alpes : 655, avenue de l'Europe - 38334 Montbonnot Saint-Ismier (France)

Unité de recherche INRIA Sophia Antipolis : 2004, route des Lucioles - BP 93 - 06902 Sophia Antipolis Cedex (France) 